\title{
Current Order and Inventory Models in Manufacturing Environments: A Review from 2008 to 2018
}

\author{
https://doi.org/10.3991/ijoe.v14i06.8055 \\ Zohreh Momeni ${ }^{\square}$, Amir Azizi \\ Islamic Azad University, Tehran, Iran \\ zohrehmomeni21@gmail.com
}

\begin{abstract}
One of the issues of designing supply chain network is "Supply chain ordering management ". Extra costs are the most important factors in the survival of an organization and have a significant impact on company competitiveness. However, holding inventory, order accepting, and functional risks are factors that have not been studied simultaneously. The purpose of this paper is to provide a review on order and inventory and use of Activity based costing approach in regard to supply chain management and costs managing. This paper selected and reviewed 56 published articles in a decade of 32 important journals related to order and inventory of supply chain manufacturing industries which chosen from the "Science direct and Scopus" databases and in this regard, the applications of the Artificial Neural Network method which called "ANN", ant colony algorithm and queue theory have been proposed. All published articles were categorized based on the author number, The first author name, publication year, problems, factors, type of manufacturing industries, research methods and results and findings. Finally, International Journal of production Economics was ranked the first. Results of this paper acknowledge that order management and inventory control can help decision makers in solving some problems under uncertainties situations of demands in environmental manufacturing industries and this approach have seen increasing interest among previous researchers to use this approach in various steps of supply chain management.
\end{abstract}

Keywords — order management, inventory control, operational risk, activitybased cost

\section{Introduction}

The supply chain management is the management of all manufacturing and supplying processes, from raw material to finished customers, that covers the whole value chain from material extraction to product lifetime. Some go further and consider recycling of raw materials in the range of the supply chain management. One of the most important features that can be mentioned in order to manage supply chain orders are: the profitability of orders, long-term orders earnings, increased customer loyalty, long-term cooperation with the company, minimizing the total costs, also it involves forward flows in order to reduce fixed and variable costs and increase customer responsiveness. Applying Dispatch volume limit, increases both the ordering cycle and 
the total annual costs. With growing up in the number of replenishment, the cost of the system will enhance. Due to gained costs in order to unnecessary redistribution, a significant leap in costs occurs. Furthermore, by increasing maintenance costs and reducing deficiency cost, the total inventory at the end of the periods is reduced. Haji and et al. studied queueing Inventory System in a Two-level Supply Chain with Onefor-One Ordering Policy. In this study, the integrated services system was used in a two-level supply chain in which it was located. Retailer only was a vendor and looking for a stock-based stock policy. Inventory system total cost by retailer is much lower than without a retailer system [4]. Tat and et al. developed Economic-OrderQuantity (EOQ) model with instantaneous deteriorating items for a vendor-managed inventory (VMI) system. In this survey, they studied the EOQ model for deteriorating items in two cases (with and without shortages) to evaluate how VMI affects supply chain. They considered two-level supply chain (single supplier and a single retailer) with one instantaneous deteriorating item. The results show that VMI works better and provides lower costs in all circumstances of traditional supply chain [10]. Zhang and et al. probed in Multi-objective optimization for sustainable supply chain network design considering multiple distribution channels. The Multi Distribution Centre Supply Chain Network (MDCSCN) model is more innovative and pioneered as it meets the latest requirements and outperforms the conventional Supply Chain Network (SCN). Realization of the paradigm changing of traditional SCN to the new one with multiple distribution channels and the complexity of information analysis, can assist management to schedule with importance of optimizing MDCSCN multiple goals by taking into account efficiency and the capacity of facilities and transportation [22]. Kumar and et al. surveyed in developing of a Novel Lot-sizing Model with Variable Lead Time in Supply Chain Environment. The main point of their results is, as the time of purchasing growth, the amount of economic order will rise too [39]. The study of literature shows that there are several types of inventory control models in multi-product environments. The total cost consists of two components:

1. Cost of preparation or ordering costs: includes the cost of adjusting machinery and facility before production in manufacturing sectors. The cost for products provided through the manufacturer consists of the cost of preparing and receiving orders and the cost of transporting goods.

2. Maintenance cost: includes the cost of maintaining the parts in the warehouse.

The cost of ordering items is also composed of two components:

a) the cost of ordering which is independent of the order quantity.

b) Variable order costs that depend on the order of the various products.

Costs are generally classified into four groups:

1. Cost of product unit operations: these costs proportional the number of goods produced, like the cost of machining time, material costs and direct wages.

2. Cost of activities related to production categories: such as management and holding inventory costs, set up of devices, and so on. 
3. Cost of specific product-related activities: such as design cost, process engineering, etc.

4. The cost of maintenance activities and management of facilities and equipment: such as rent, utilization, reparation and maintenance costs.

This review paper aims to provide review of current order and inventory models due to costs problems. A wide variety of previous studies reviewed the order management or inventory control in supply chain problems such as inventory control problem of logistical systems but there are several studies that survey in service systems for instance bank as case study. The remainder of this paper is organized as follows. Section 1 provides an overview of literature of current surveys in order and inventory. Section 2 several critical factors of the researches were highlighted. Section 3 of this paper attempted to discuss on the obtain findings and results and Section 4 provides some conclude remarks, limitations of this study, and suggestions for future researches.

\section{Literature review}

In literature review section, we highlight some important factors that are as follows:

- Extra buffer stock required to eliminate stock out

- Unit cost of time

- Cost of carrying inventory in percentage per year

- Ordering cost in per order

- Demand in lead time

- Number of stock units demanded in time period

- Distribution of demand in lead time

- Procurement lead time

- Minimum stock in any time period

- Number of order per year

- Order quantity

- Economic order quantity

- Re-order point

- Total lead time

- Loss per unit inventory if there is no demand

- Probability of stock out in a cycle (order) [39].

- Set-up cost for supplier to produce component

- Set-up cost for plant to produce product

- Set-up cost for Distribution Centre (DC) to deliver product

- Capacity of plant to design product

- Capacity of supplier to design component

- Unit transportation cost from supplier to plant for component

- Unit transportation cost from plant to DC for product

- Unit purchasing cost of component from supplier 
- Unit production cost of product at plant

- Unit cost of throughput

- Maximum production capacity of supplier for component

- Maximum production capacity of plant for product

- Total production capacity of plant

- Minimum throughput

- Quantity of component used in one unit of product

- Service level of supplier

- Service level of plant

- Service level of DC

- Volume of product produced at plant

- Volume of product received at DC

- Volume of component provided by supplier

- Volume of product transferred from plant to DC

- Volume of component transferred from supplier to plant [19].

- Major operation costs share in general operation costs

- Number of identified activity segments

- Share of supply costs in general operation costs [23].

In addition, some critical problems that we mention that are as follows:

Investigating internal interrelationships and provide insights into the operational dynamics of single supply chain enterprises. To better focusing risk monitoring and risk management in the automotive industry supply chains on risks in order to enhance decision making in the upstream supply chain [17]. Taking into consideration the uncertainty of demand, cost of production, allocation of the transportation cost, shortage loss, tax rates and limitation of markdown rates [29]. Cost calculation for more accurate cost information than the traditional volume-based costing (VBC). using $\mathrm{ABC}$ approach with two stages to allocate and calculate the manufacturing cost which is based on resources expired of process activities [33]. Sustainable supplier selection and order allocation problem under operational and disruption risk [42], considering different shortage situations [45], minimizing the average total inventory cost [48], risk assessment of existing production units considering availability and human safety criteria [50], deciding the logistics service integrator regarding the location of the customer order decoupling point [52], analyzing the benefits of horizontal collaboration related to perishability, from transportation operations and logistics costs in the Inventory Routing Problem (IRP) with multiple suppliers and customers by developing a decision support model that can address these concerns [55], designing a resilient hub network under operational and disruption risks [56].

Furthermore, all published articles were categorized based on the author number, The first author name, publication year, problems, factors, type of manufacturing industries, research methods and results and findings. Some manufacturing industries that are surveyed in articles as a case study or collocated data from them, include bicycle, nylon plastic and refrigerator manufacturing, façade components, carpet manufacturing facility, automotive supply chain, coal mining enterprises, gas industry, locomotives railways, steel and glass company and energy production units. The 
methods that are used more than others encompass mathematical model, simulation and heuristic algorithm. Study flowchart for the identification and included of articles that are surveyed is shown in Fig. 1. In addition, more information about articles that are investigated in details is exhibited in Table 1.

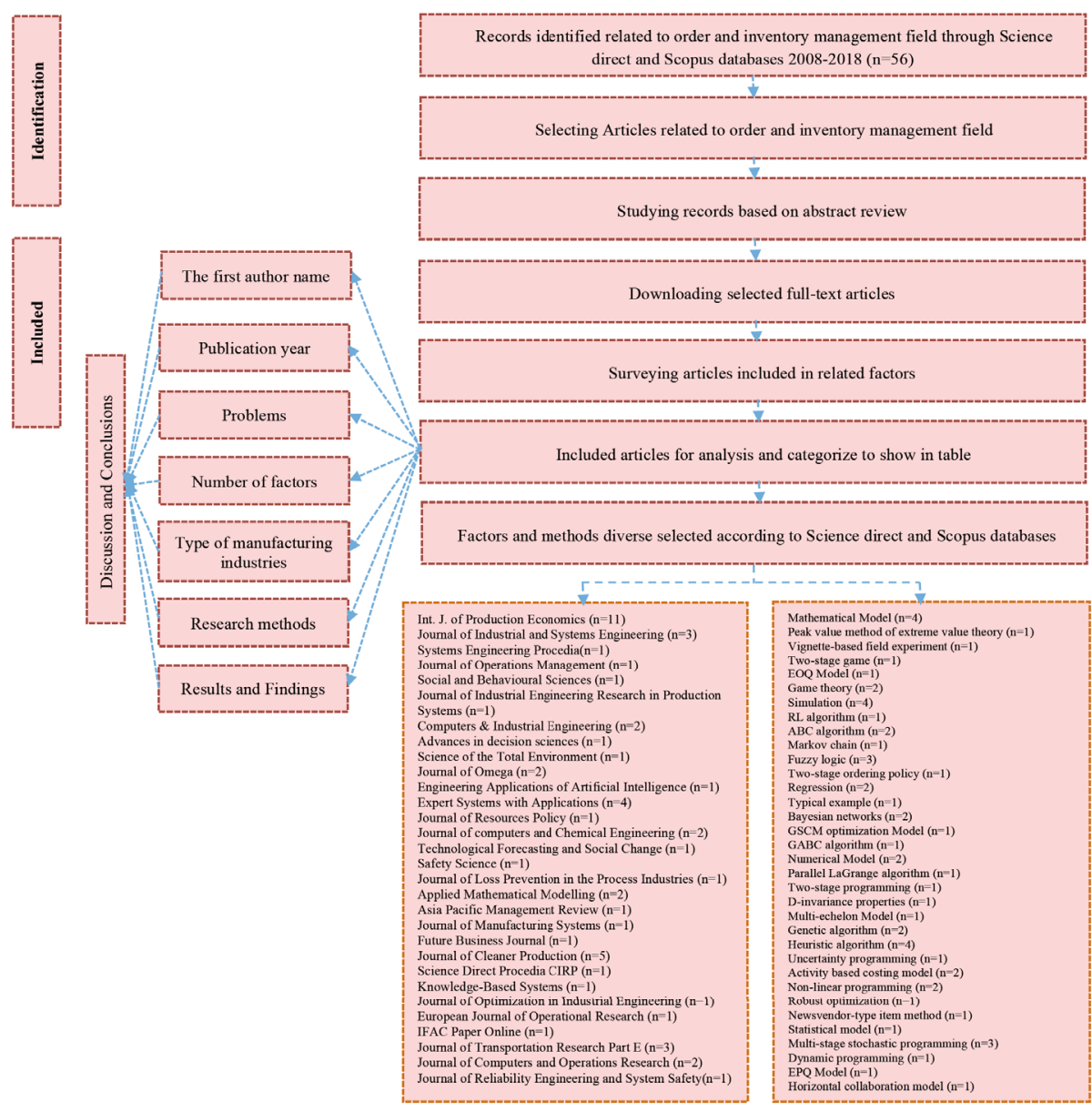

Fig. 1. Study flowchart for the identification and included of articles. 
Review-Current Order and Inventory Models in Manufacturing Environments: A Review from 2008 to...

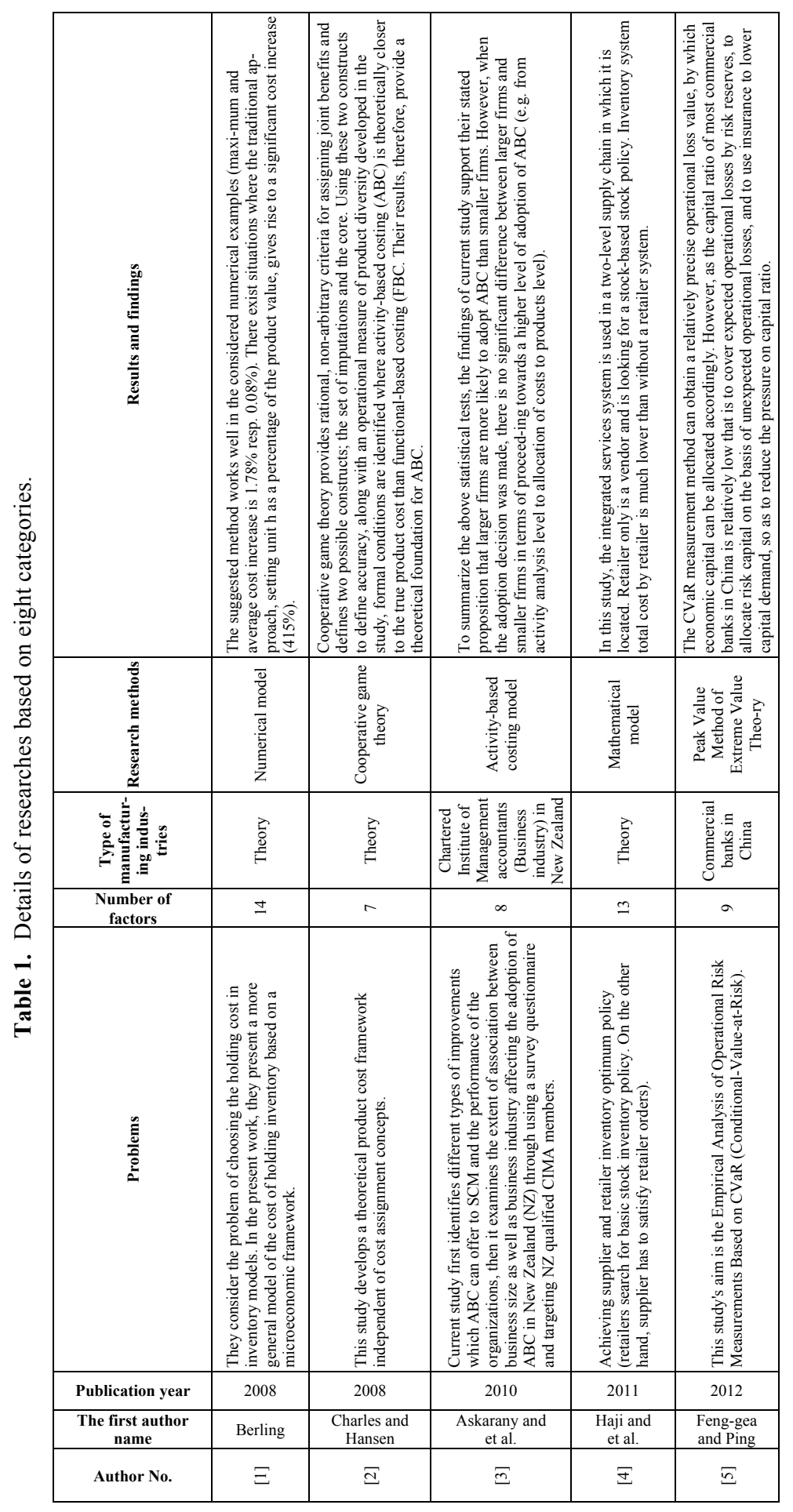


Review-Current Order and Inventory Models in Manufacturing Environments: A Review from 2008 to...

\begin{tabular}{|c|c|c|c|c|c|}
\hline 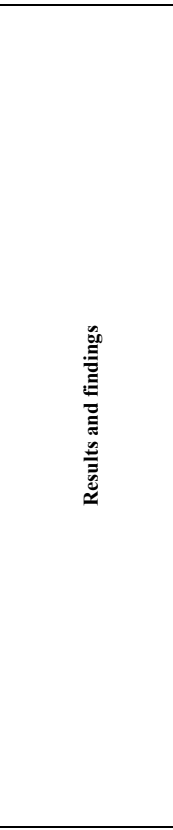 & 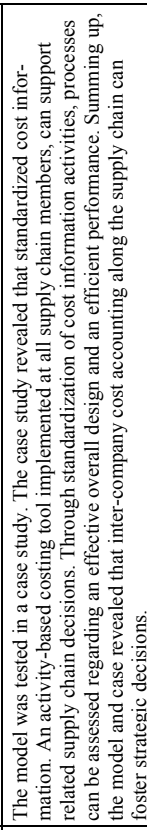 & 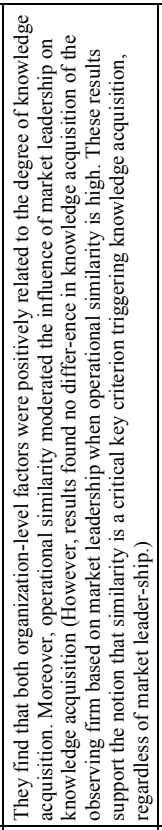 & 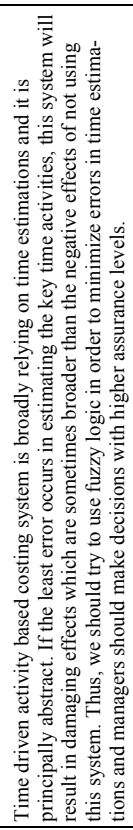 & 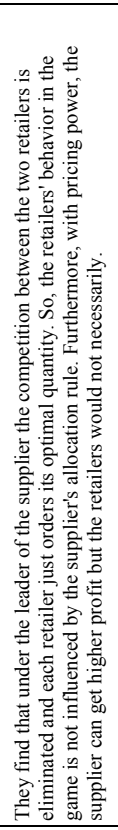 & 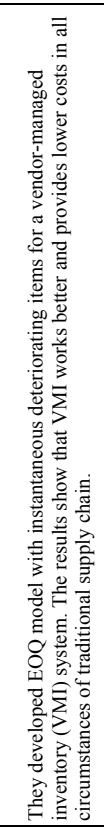 \\
\hline 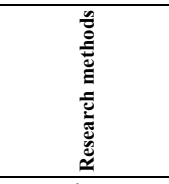 & 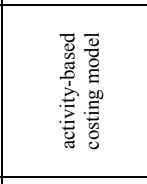 & 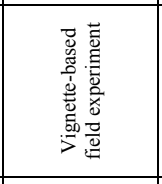 & $\frac{\sqrt{5}}{2}$ & 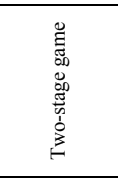 & 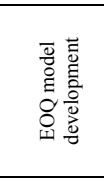 \\
\hline 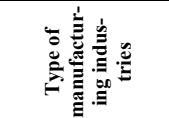 & 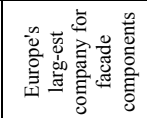 & 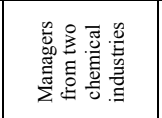 & 客 & 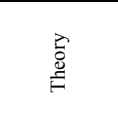 & 窘 \\
\hline $\begin{array}{c}\text { Number of } \\
\text { factors }\end{array}$ & m & $\simeq$ & $\infty$ & $\stackrel{\circ}{\simeq}$ & $\pi$ \\
\hline 這 & 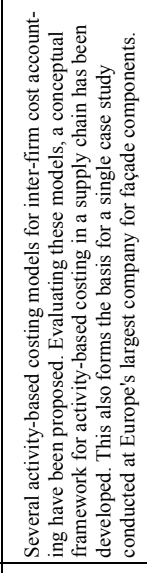 & 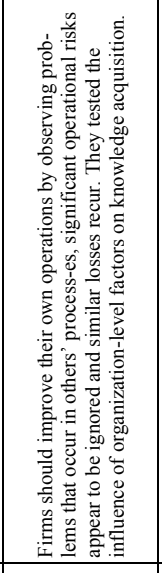 & 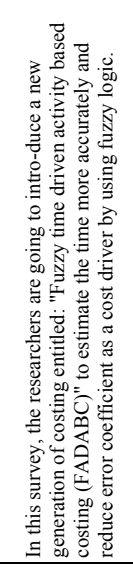 & 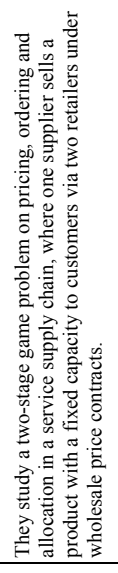 & 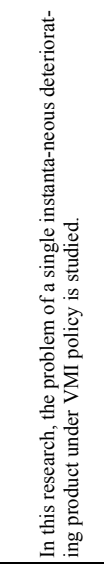 \\
\hline Publication year & 2012 & 2013 & 2013 & 2013 & 2014 \\
\hline $\begin{array}{c}\text { The first author } \\
\text { name }\end{array}$ & $\begin{array}{c}\text { Schulze and } \\
\text { et al. }\end{array}$ & $\begin{array}{c}\text { Hora and } \\
\text { Klassen }\end{array}$ & $\begin{array}{l}\begin{array}{c}\text { Alinezhad } \\
\text { and et al. }\end{array} \\
\end{array}$ & $\begin{array}{l}\text { Wei and } \\
\text { et al. }\end{array}$ & $\begin{array}{c}\text { Tat and et } \\
\text { al. } \\
\text { al }\end{array}$ \\
\hline Author No. & $\underline{6}$ & E & 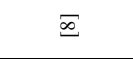 & $\Xi$ & $\Xi$ \\
\hline
\end{tabular}


Review - Current Order and Inventory Models in Manufacturing Environments: A Review from 2008 to...

\begin{tabular}{|c|c|c|c|c|c|}
\hline 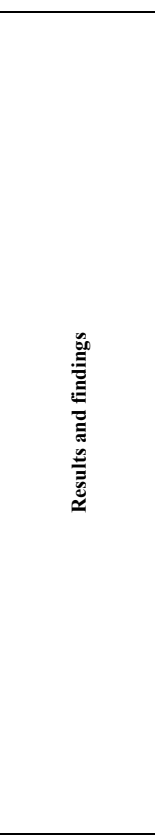 & 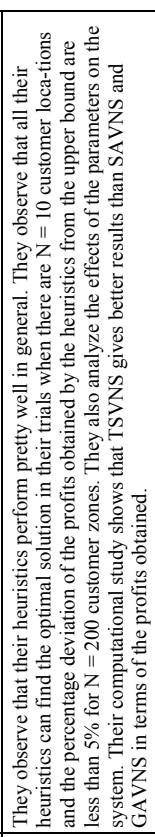 & 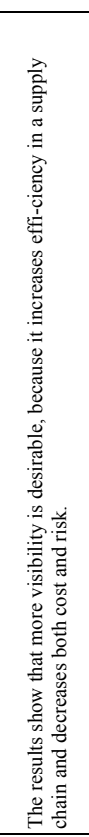 & 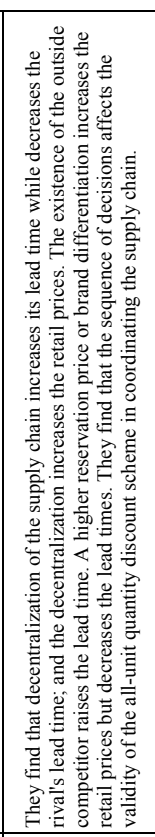 & 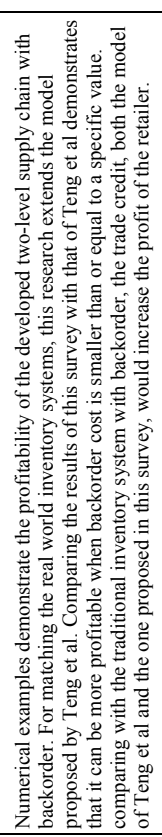 & 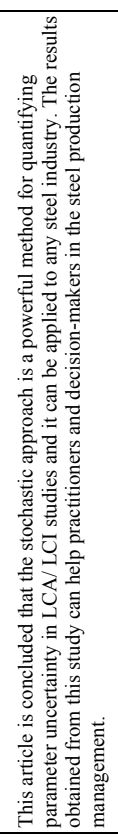 \\
\hline 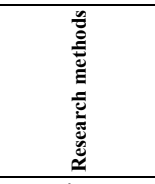 & 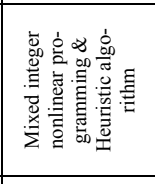 & 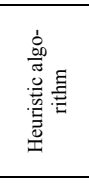 & 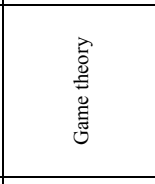 & 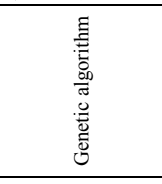 & 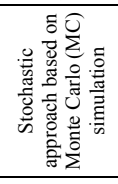 \\
\hline 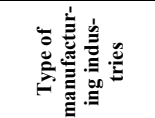 & 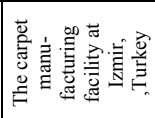 & 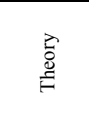 & 客 & 麇 & 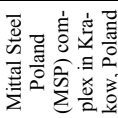 \\
\hline $\begin{array}{c}\text { Number of } \\
\text { factors }\end{array}$ & \pm & \pm & 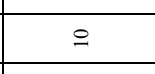 & \pm & $r$ \\
\hline 量 & 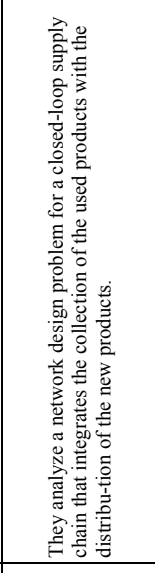 & 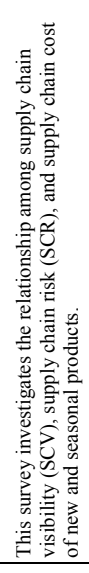 & 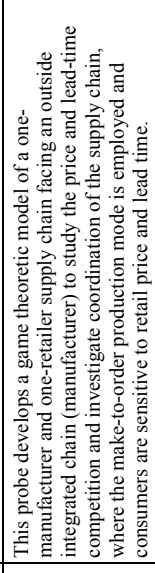 & 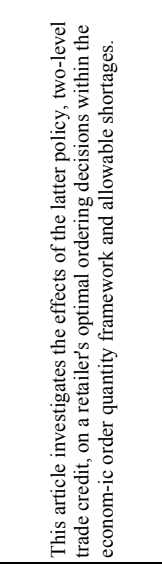 & 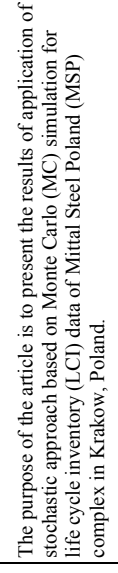 \\
\hline Publication year & 2014 & 2014 & 2014 & 2014 & 2014 \\
\hline $\begin{array}{c}\text { The first author } \\
\text { name }\end{array}$ & $\begin{array}{l}\text { Kazemza- } \\
\text { deh and et }\end{array}$ & $\begin{array}{l}\begin{array}{l}\text { eand } \\
\text { Mellat }\end{array} \\
\end{array}$ & $\begin{array}{c}\text { Xiao and } \\
\text { Chen } \\
\end{array}$ & $\begin{array}{l}\text { Molamoham- } \\
\text { adi and et al. }\end{array}$ & $\begin{array}{l}\begin{array}{c}\text { Bieda } \\
\text { and et al. }\end{array} \\
\text { and }\end{array}$ \\
\hline Author No. & $\Xi$ & $\bar{\Xi}$ & $\stackrel{m}{3}$ & $\Xi$ & $\underline{n}$ \\
\hline
\end{tabular}


Review-Current Order and Inventory Models in Manufacturing Environments: A Review from 2008 to...

\begin{tabular}{|c|c|c|c|c|c|}
\hline 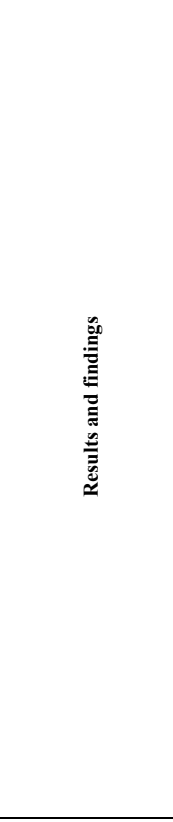 & 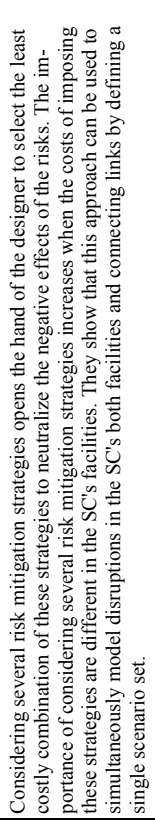 & 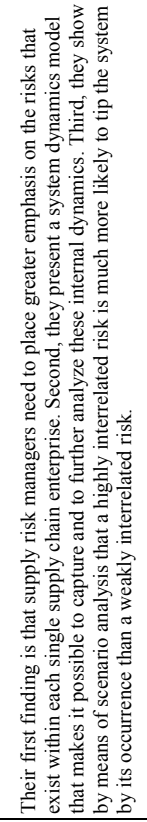 & 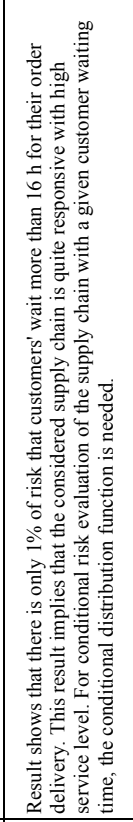 & 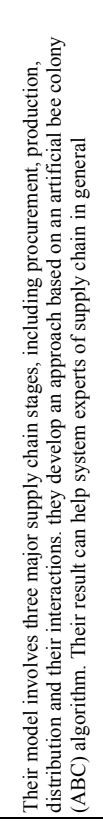 & 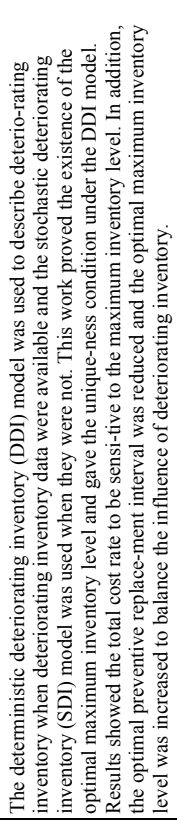 \\
\hline 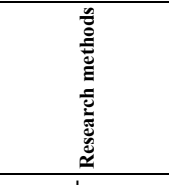 & 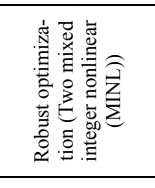 & 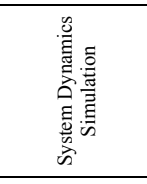 & 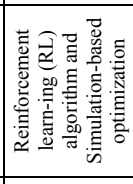 & 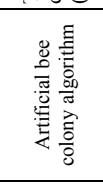 & 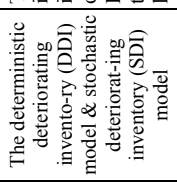 \\
\hline 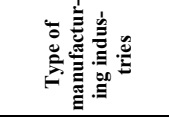 & 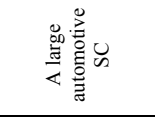 & 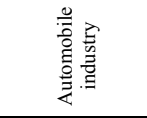 & 产 & 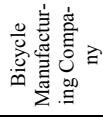 & 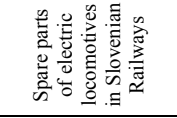 \\
\hline $\begin{array}{c}\text { Number of } \\
\text { factors }\end{array}$ & o & 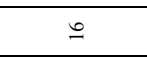 & r & d & $\dot{m}$ \\
\hline 号 & 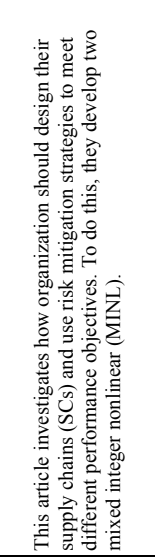 & 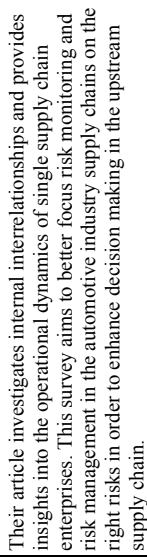 & 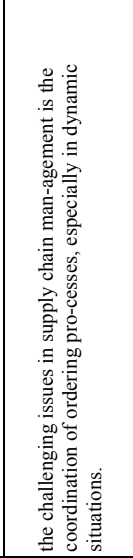 & 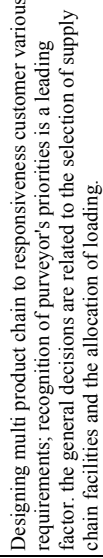 & 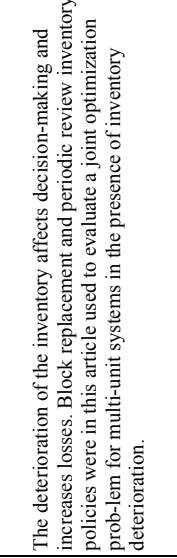 \\
\hline Publication year & 2015 & 2015 & 2015 & 2015 & 2015 \\
\hline $\begin{array}{c}\text { The first author } \\
\text { name }\end{array}$ & $\begin{array}{l}\text { Mohammad } \\
\text { dust and et al. }\end{array}$ & $\begin{array}{c}\begin{array}{c}\text { Guertler } \\
\text { and Spinler }\end{array} \\
\end{array}$ & vi and et & $\begin{array}{l}\text { and et } \\
\text { and }\end{array}$ & Jiang and et al. \\
\hline Author No. & $\stackrel{\underline{G}}{\Xi}$ & $\Xi$ & $\stackrel{\infty}{=}$ & $\Xi$ & $\bar{\Xi}$ \\
\hline
\end{tabular}


Review - Current Order and Inventory Models in Manufacturing Environments: A Review from 2008 to...

\begin{tabular}{|c|c|c|c|c|c|}
\hline 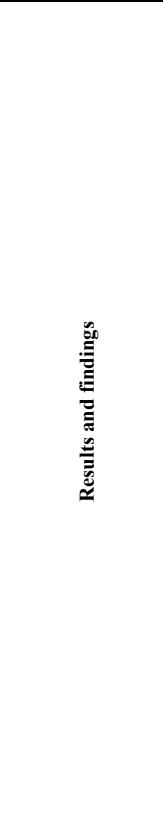 & 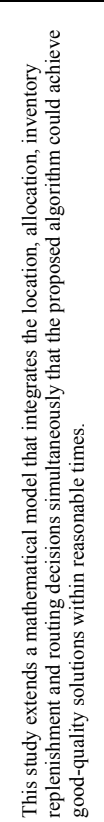 & 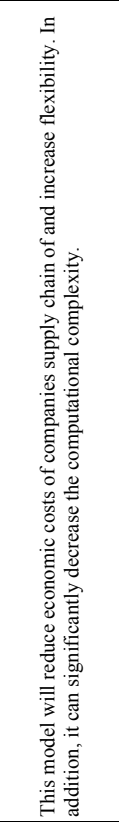 & 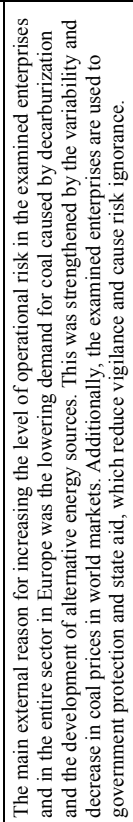 & 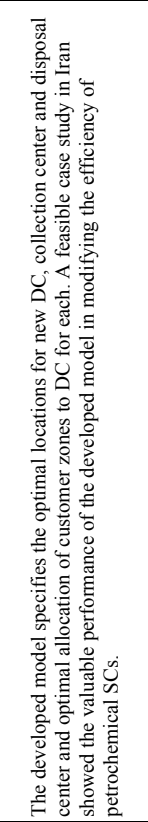 & 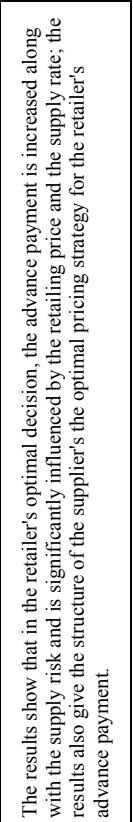 \\
\hline 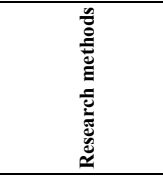 & 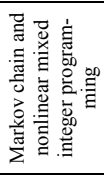 & 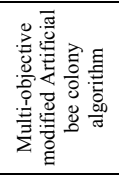 & 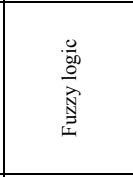 & 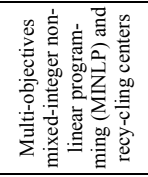 & 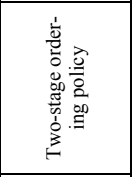 \\
\hline 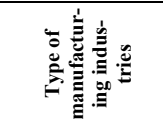 & 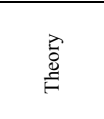 & 毞 & 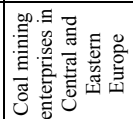 & 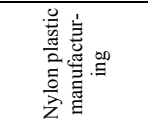 & 空 \\
\hline $\begin{array}{c}\text { Number of } \\
\text { factors }\end{array}$ & $\simeq$ & \pm & 并 & 3 & $\stackrel{n}{=}$ \\
\hline 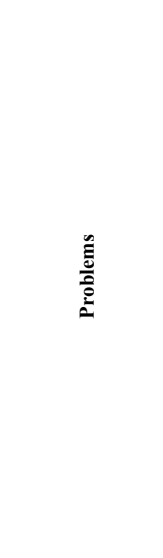 & 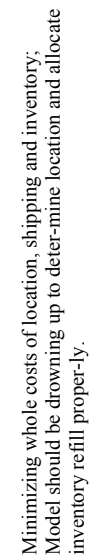 & 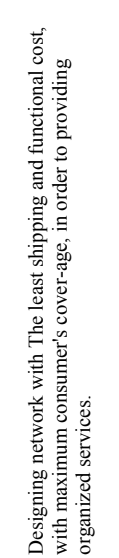 & 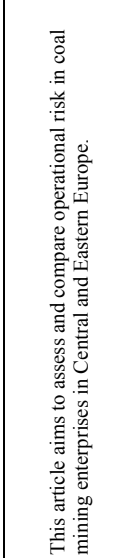 & 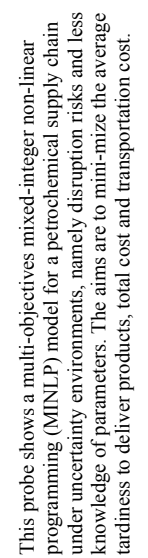 & 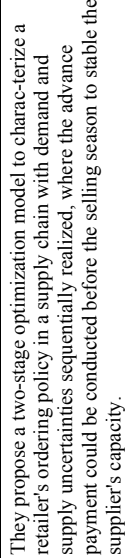 \\
\hline Publication y & 2016 & 2016 & 2016 & 2016 & 2016 \\
\hline $\begin{array}{c}\begin{array}{c}\text { The first author } \\
\text { name }\end{array} \\
\end{array}$ & $\begin{array}{l}\text { ihan } \\
\text { and et }\end{array}$ & $\begin{array}{l}\begin{array}{l}\text { Zhang } \\
\text { and et al. }\end{array} \\
\text { and }\end{array}$ & $\begin{array}{l}\text { and } \\
\text { Jonek- }\end{array}$ & $\begin{array}{l}\begin{array}{l}\text { Yousefif Baba- } \\
\text { dia and et al. }\end{array} \\
\text { dit. }\end{array}$ & $\begin{array}{l}\begin{array}{c}\text { Zhang } \\
\text { and et al. }\end{array} \\
\text { and }\end{array}$ \\
\hline Author No. & $\overline{\mathrm{a}}$ & $\bar{d}$ & $\bar{a}$ & 志 & $=$ \\
\hline
\end{tabular}


Review - Current Order and Inventory Models in Manufacturing Environments: A Review from 2008 to...

\begin{tabular}{|c|c|c|c|c|c|}
\hline 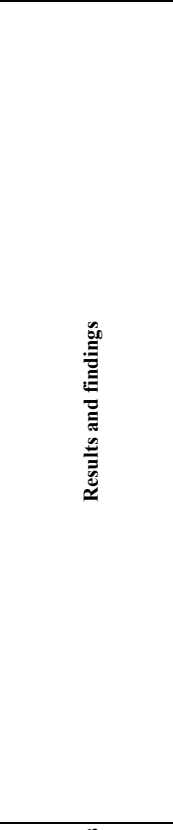 & 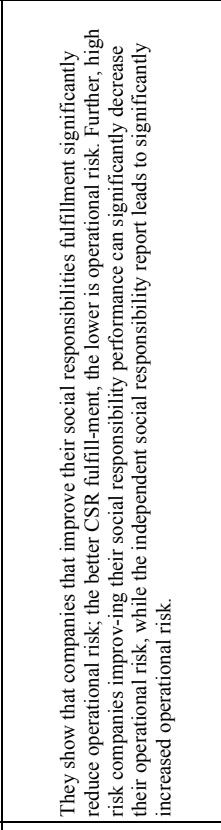 & 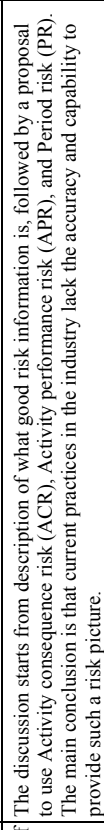 & 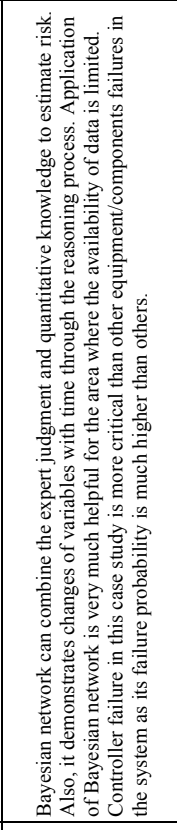 & 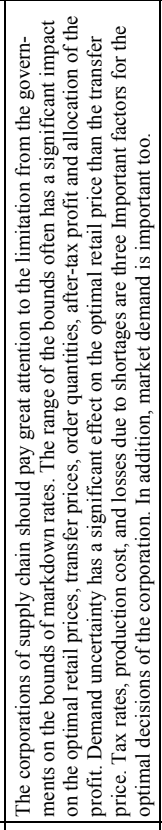 & 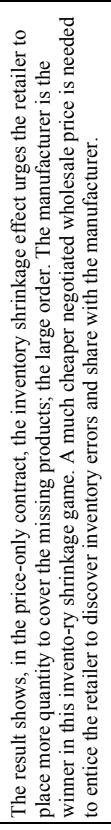 \\
\hline 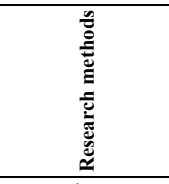 & 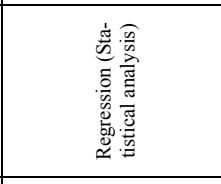 & 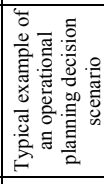 & 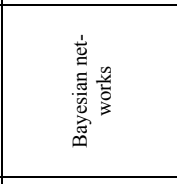 & 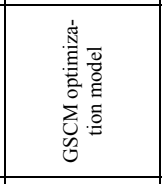 & 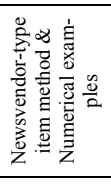 \\
\hline 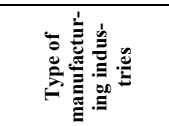 & 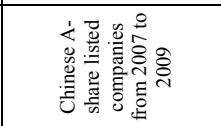 & 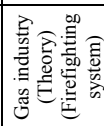 & 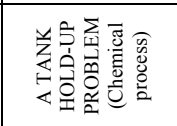 & 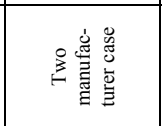 & 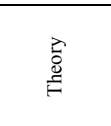 \\
\hline $\begin{array}{c}\text { Number of } \\
\text { factors }\end{array}$ & \pm & $a$ & $r$ & 2 & $\simeq$ \\
\hline 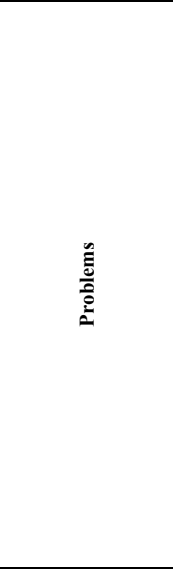 & 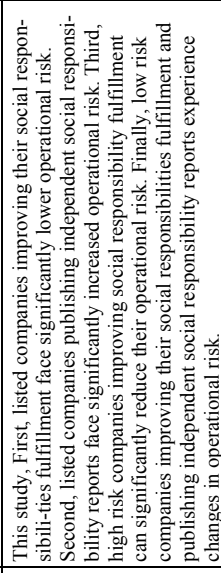 & 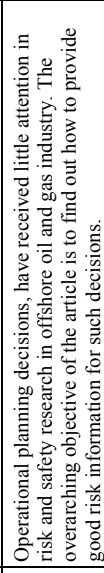 & 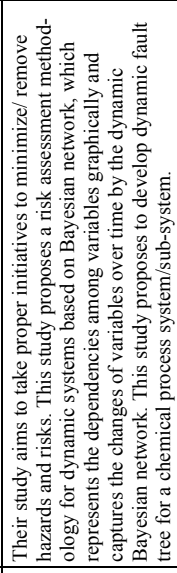 & 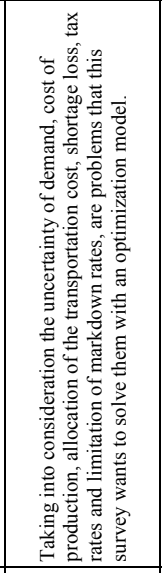 & 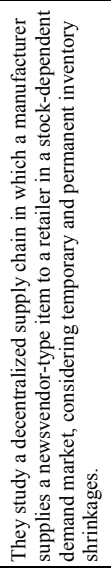 \\
\hline Publication year & 2016 & 2016 & 2016 & 2016 & 2016 \\
\hline $\begin{array}{c}\text { The first author } \\
\text { name }\end{array}$ & Zhao and et al. & $\begin{array}{l}\text { and } \\
\text { Hauge } \\
\end{array}$ & $\begin{array}{c}\text { Barua and et } \\
\text { al. }\end{array}$ & $\begin{array}{c}\text { Zhang and } \\
\text { et al. }\end{array}$ & $\begin{array}{c}\text { Wang and } \\
\text { etal. }\end{array}$ \\
\hline Author No. & ¿ & ¿ & 可 & ฮิ & 㧀 \\
\hline
\end{tabular}


Review - Current Order and Inventory Models in Manufacturing Environments: A Review from 2008 to...

\begin{tabular}{|c|c|c|c|c|c|c|}
\hline 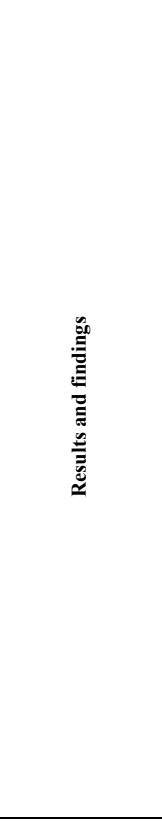 & 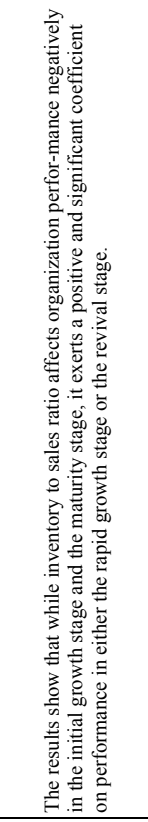 & 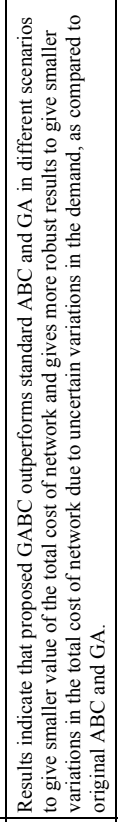 & 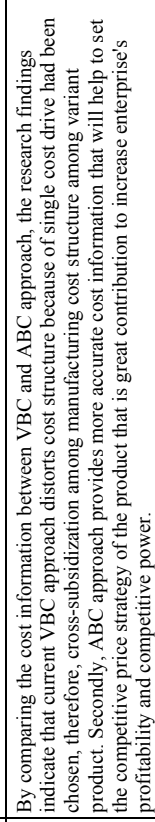 & 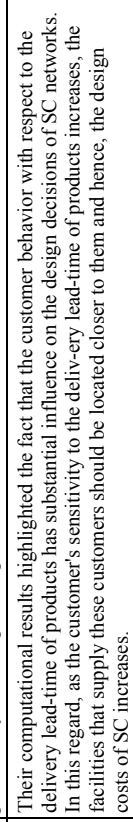 & 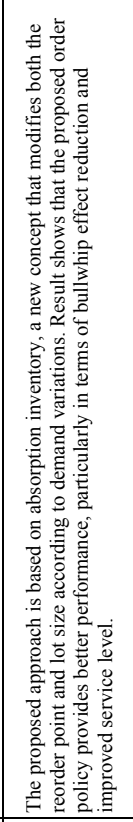 & 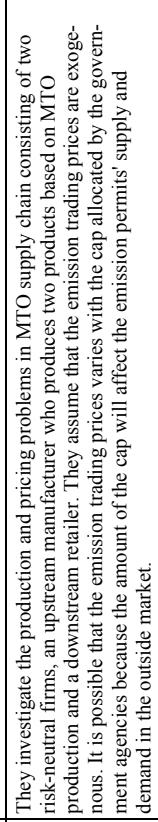 \\
\hline 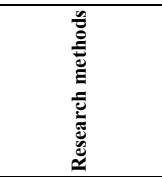 & 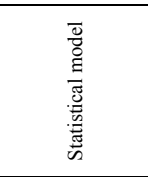 & 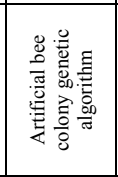 & 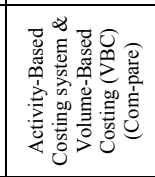 & 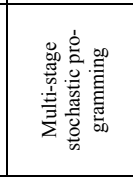 & 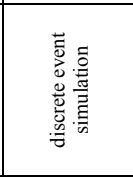 & 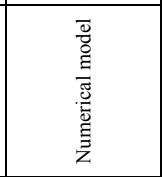 \\
\hline 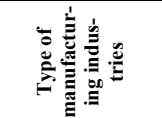 & 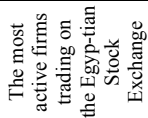 & 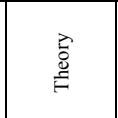 & 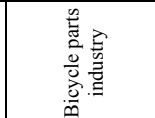 & 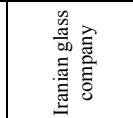 & 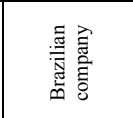 & 害 \\
\hline $\begin{array}{c}\text { Number of } \\
\text { factors }\end{array}$ & 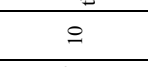 & $\bar{m}$ & 0 & a & \pm & 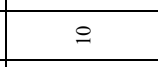 \\
\hline 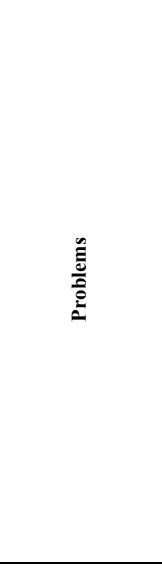 & 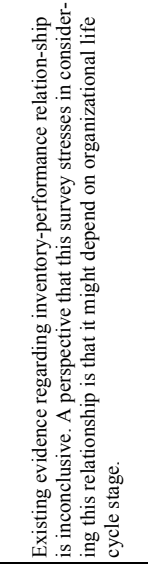 & 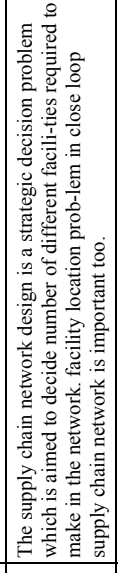 & 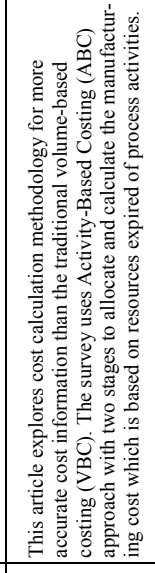 & 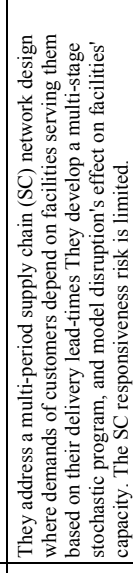 & 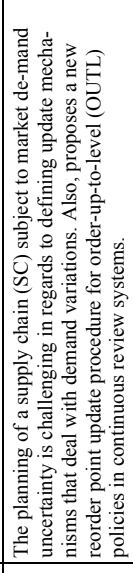 & 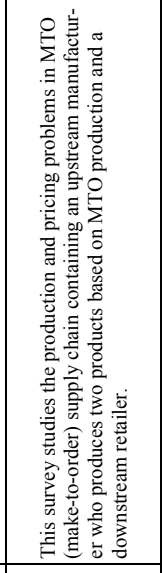 \\
\hline Publication year & 2016 & 2017 & 2017 & 2017 & 2017 & 2017 \\
\hline $\begin{array}{c}\text { The first author } \\
\text { name }\end{array}$ & $\begin{array}{c}\begin{array}{c}\text { Elsayedn } \\
\text { and Wahba }\end{array} \\
\end{array}$ & $\begin{array}{l}\text { cul } \\
\text { and et }\end{array}$ & Lu and et al. & $\begin{array}{l}\text { Fattahi } \\
\text { and et al. }\end{array}$ & $\begin{array}{l}\text { Pacheco } \\
\text { and et al. }\end{array}$ & $\begin{array}{c}\mathrm{Xu} \text { and et } \\
\text { al. }\end{array}$ \\
\hline Author No. & $\bar{m}$ & $\sqrt[ల ్]{0}$ & 胥 & F & 贾 & 宮 \\
\hline
\end{tabular}


Review-Current Order and Inventory Models in Manufacturing Environments: A Review from 2008 to...

\begin{tabular}{|c|c|c|c|c|}
\hline 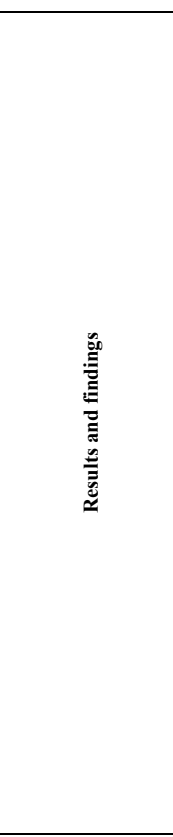 & 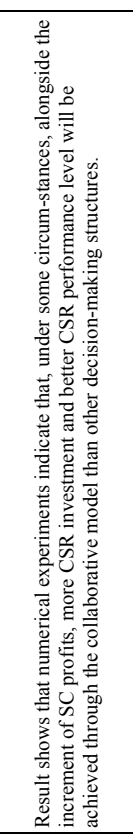 & 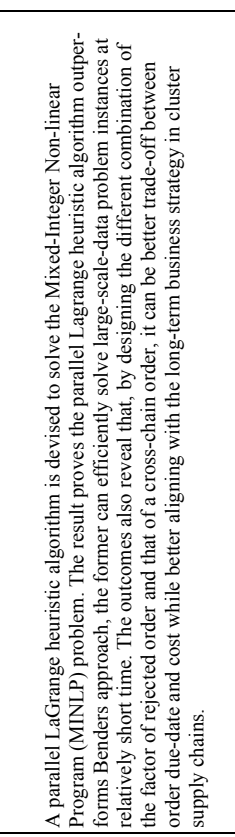 & 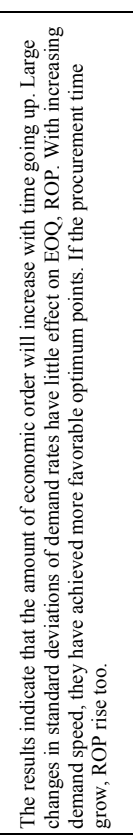 & 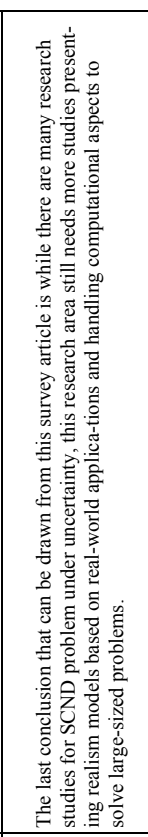 \\
\hline 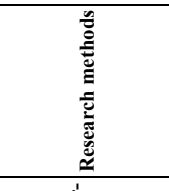 & 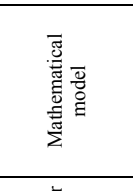 & 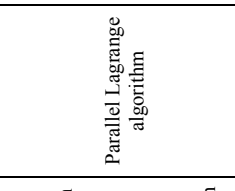 & 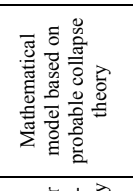 & 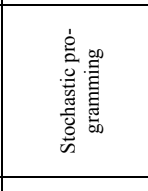 \\
\hline 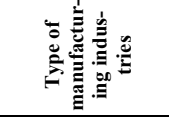 & 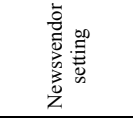 & 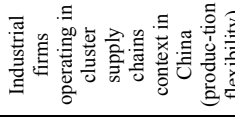 & 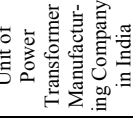 & 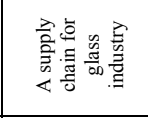 \\
\hline $\begin{array}{c}\text { Number of } \\
\text { factors }\end{array}$ & $\simeq$ & $\stackrel{\circ}{\circ}$ & 4 & $\stackrel{\infty}{\infty}$ \\
\hline 這 & 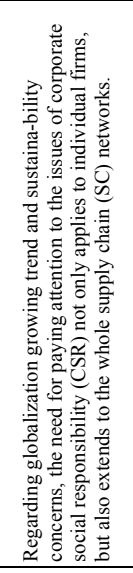 & 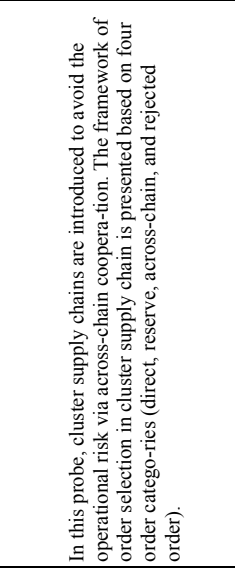 & 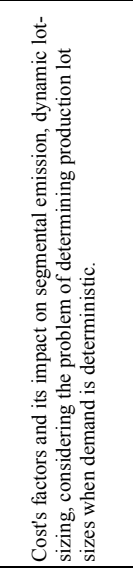 & 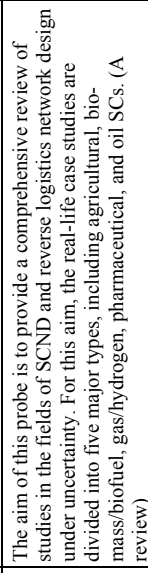 \\
\hline Publication year & 2017 & 2017 & 2017 & 2017 \\
\hline $\begin{array}{c}\text { The first author } \\
\text { name }\end{array}$ & $\begin{array}{c}\text { Nematollahia } \\
\text { and et al. }\end{array}$ & Li and et al. & $\begin{array}{c}\text { Kumar and } \\
\text { et al. }\end{array}$ & $\begin{array}{l}\begin{array}{l}\text { Govindan } \\
\text { and et al. }\end{array} \\
\end{array}$ \\
\hline Author No. & $\sqrt{0}$ & $\underset{\infty}{\infty}$ & $\bar{\rho}$ & 守 \\
\hline
\end{tabular}


Review-Current Order and Inventory Models in Manufacturing Environments: A Review from 2008 to...

\begin{tabular}{|c|c|c|c|c|}
\hline 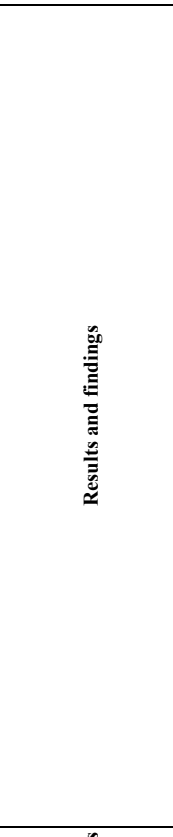 & 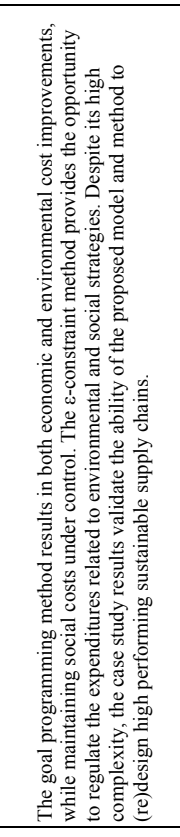 & 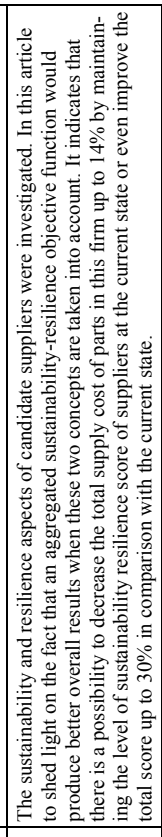 & 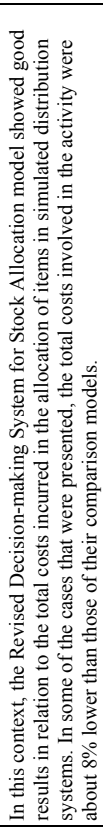 & 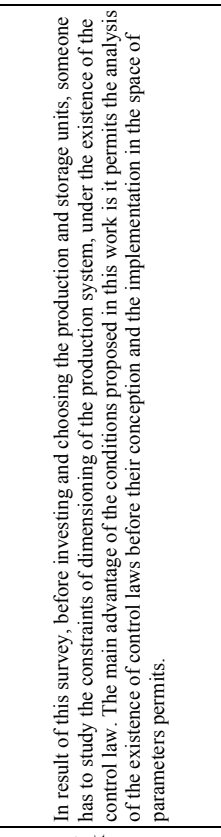 \\
\hline 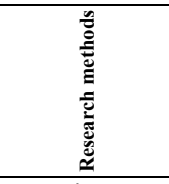 & 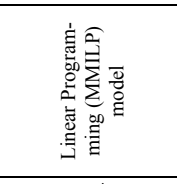 & 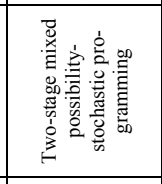 & 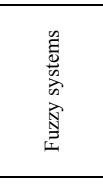 & 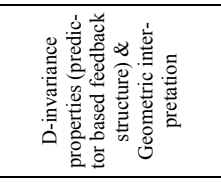 \\
\hline 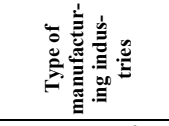 & 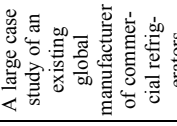 & 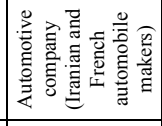 & 惫 & 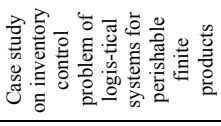 \\
\hline $\begin{array}{c}\text { Number of } \\
\text { factors }\end{array}$ & $:$ & i & $\stackrel{\infty}{\sim}$ & 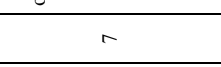 \\
\hline 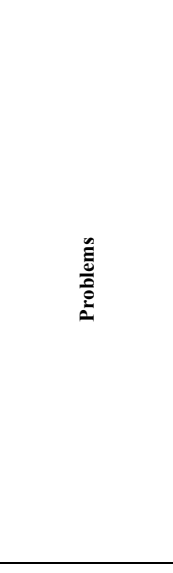 & 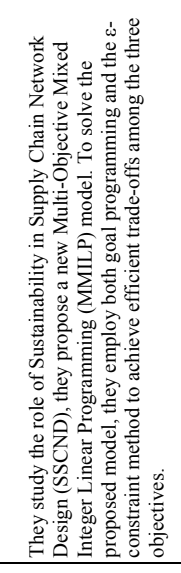 & 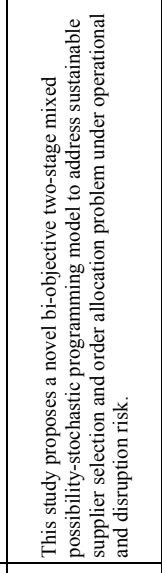 & 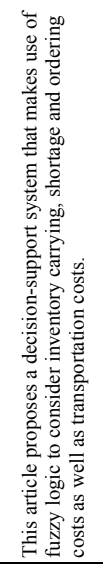 & 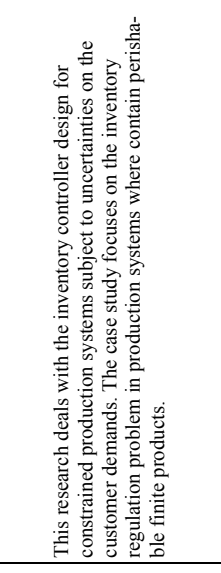 \\
\hline Publication year & 2017 & 2018 & 2017 & 2017 \\
\hline $\begin{array}{c}\text { The first author } \\
\text { name }\end{array}$ & $\begin{array}{l}\text { Arampantzi and } \\
\text { et al. }\end{array}$ & $\begin{array}{c}\text { Vahidi and } \\
\text { et al. }\end{array}$ & $\begin{array}{l}\begin{array}{c}\text { Wanke } \\
\text { and et al. }\end{array} \\
\text { and }\end{array}$ & Abbou and et al. \\
\hline Author No. & 王 & $\bar{z}$ & $\underline{\underline{9}}$ & 军 \\
\hline
\end{tabular}


Review-Current Order and Inventory Models in Manufacturing Environments: A Review from 2008 to...

\begin{tabular}{|c|c|c|c|c|}
\hline 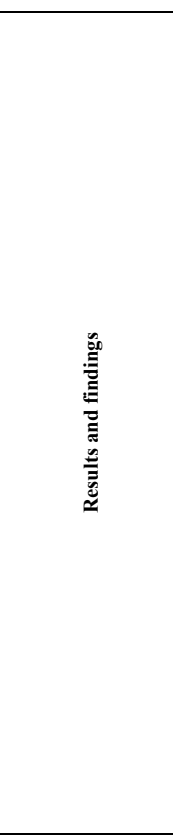 & 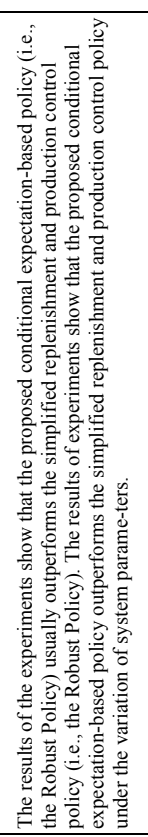 & 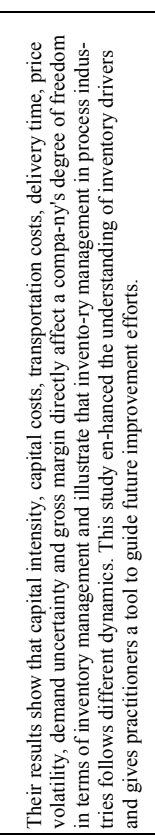 & 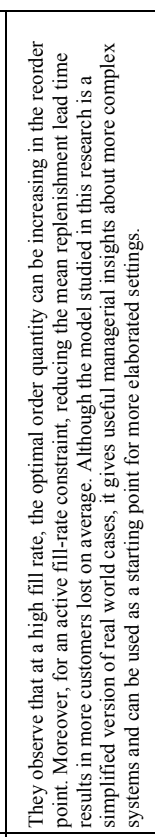 & 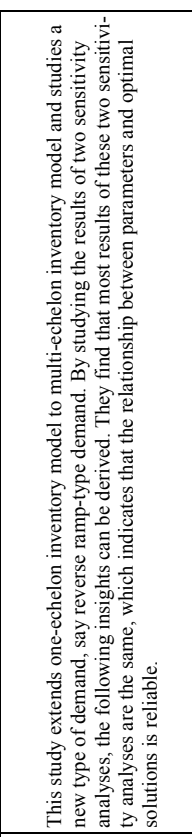 \\
\hline 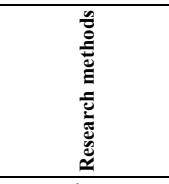 & 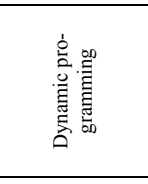 & 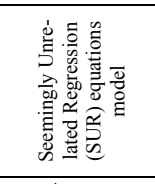 & 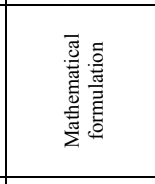 & 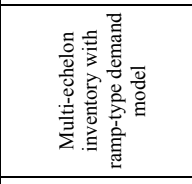 \\
\hline 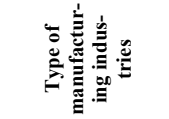 & 彦 & 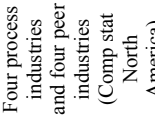 & 荵 & 롤 \\
\hline $\begin{array}{c}\text { Number of } \\
\text { factors }\end{array}$ & $\bar{a}$ & $=$ & $a$ & o \\
\hline 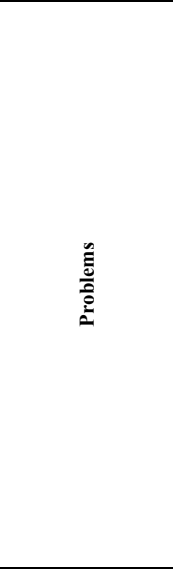 & 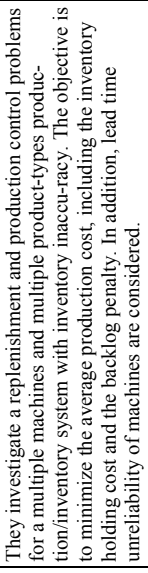 & 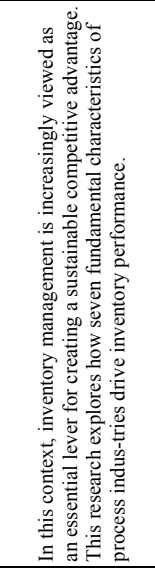 & 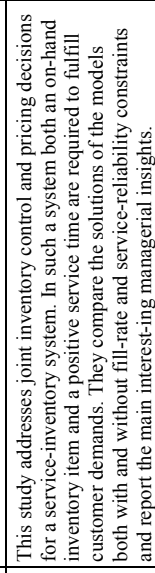 & 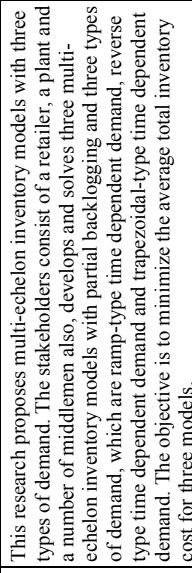 \\
\hline Publication year & 2017 & 2017 & 2017 & 2017 \\
\hline $\begin{array}{c}\text { The first author } \\
\text { name }\end{array}$ & $\begin{array}{c}\begin{array}{c}\mathrm{L} \text { a and } \\
\text { Wang }\end{array} \\
\end{array}$ & $\begin{array}{c}\text { Moser and et } \\
\text { al. }\end{array}$ & $\begin{array}{c}\text { Marand and } \\
\text { et al. }\end{array}$ & Daia and et al. \\
\hline Author No. & 垔 & 昌 & 导 & 垔 \\
\hline
\end{tabular}


Review - Current Order and Inventory Models in Manufacturing Environments: A Review from 2008 to...

\begin{tabular}{|c|c|c|c|c|c|}
\hline 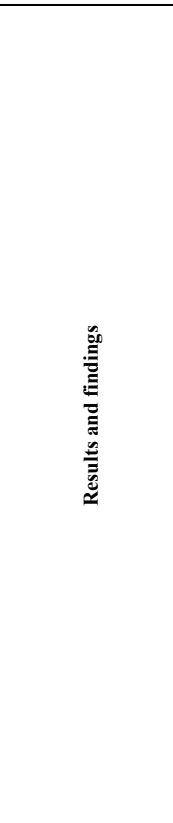 & 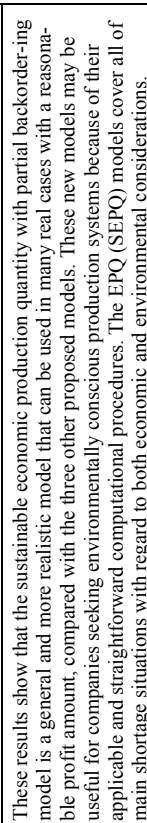 & 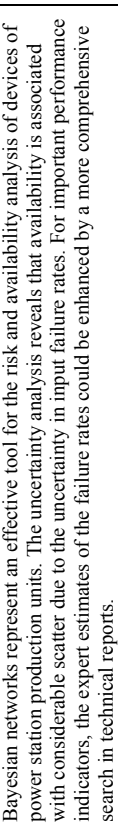 & 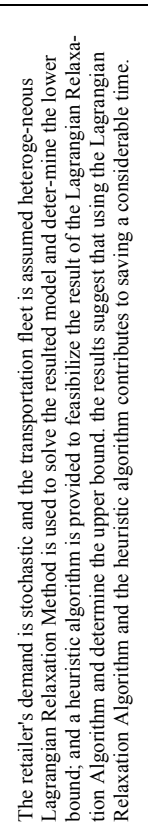 & 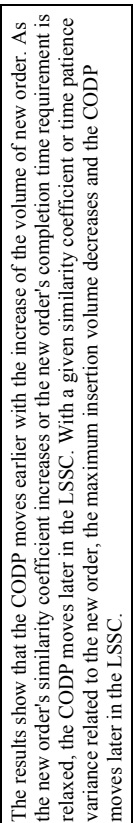 & 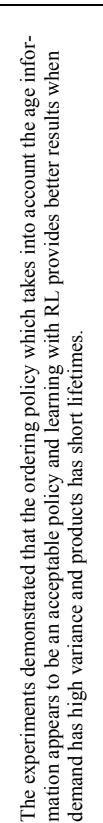 \\
\hline 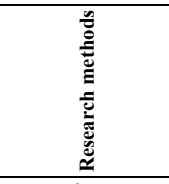 & 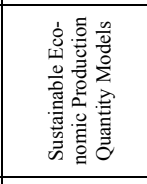 & 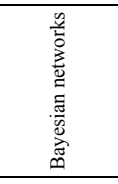 & 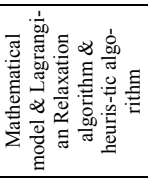 & 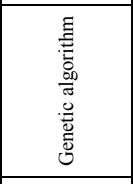 & 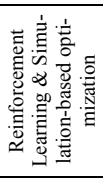 \\
\hline 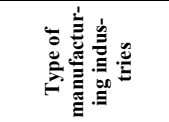 & 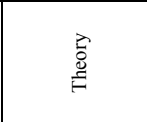 & 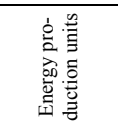 & $\begin{array}{l}\text { : } \\
\text { d }\end{array}$ & 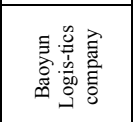 & 突 \\
\hline $\begin{array}{c}\text { Number of } \\
\text { factors }\end{array}$ & m & $r$ & 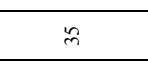 & क & + \\
\hline 旁 & 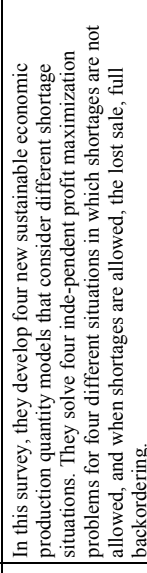 & 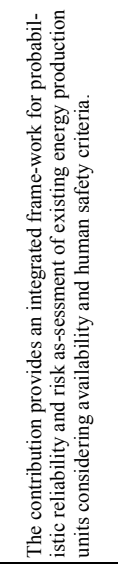 & 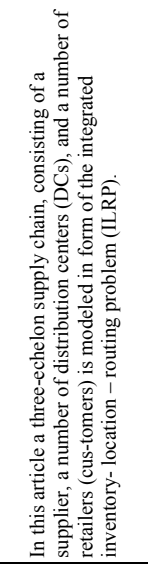 & 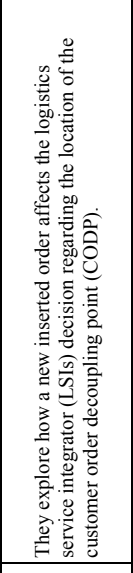 & 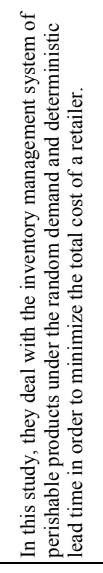 \\
\hline Publication year & 2018 & 2018 & 2018 & 2018 & 2018 \\
\hline $\begin{array}{c}\text { The first author } \\
\text { name }\end{array}$ & $\begin{array}{l}\begin{array}{l}\text { Taleizadeh } \\
\text { and et al. }\end{array} \\
\end{array}$ & $\begin{array}{l}\text { Sykora } \\
\text { and et al. }\end{array}$ & $\begin{array}{l}\text { Rafie-Majd } \\
\text { and et al. }\end{array}$ & $\begin{array}{l}\text { Liu and } \\
\text { et al. }\end{array}$ & $\begin{array}{c}\text { Kara and } \\
\text { Dogan }\end{array}$ \\
\hline Author No. & 豆 & $\overline{\check{n}}$ & $\overline{\bar{n}}$ & त्रू & $\overline{\tilde{n}}$ \\
\hline
\end{tabular}


Review - Current Order and Inventory Models in Manufacturing Environments: A Review from 2008 to...

\begin{tabular}{|c|c|c|}
\hline 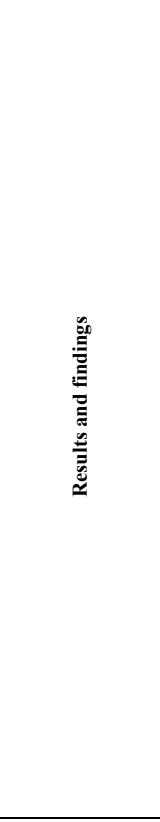 & 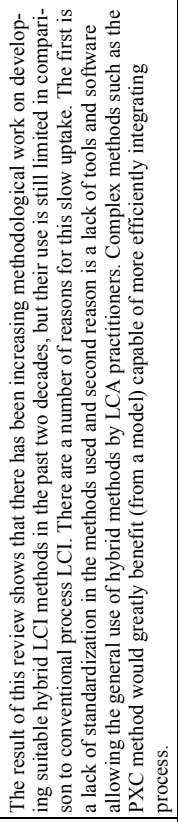 & 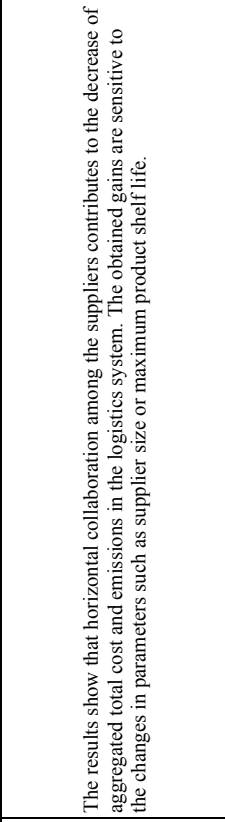 \\
\hline 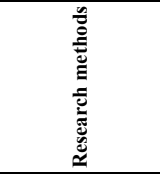 & 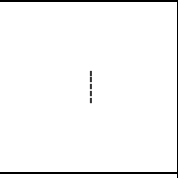 & 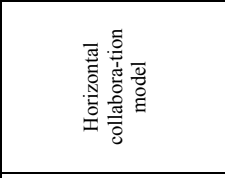 \\
\hline 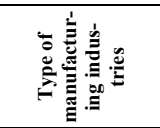 & 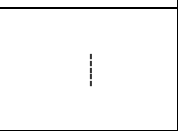 & 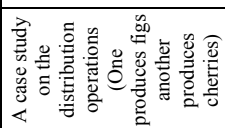 \\
\hline $\begin{array}{c}\begin{array}{c}\text { Number of } \\
\text { factors }\end{array} \\
\end{array}$ & : & 声 \\
\hline 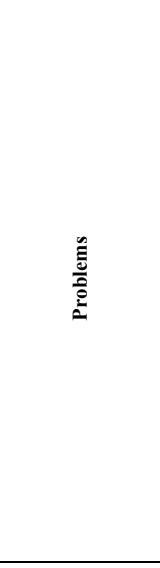 & 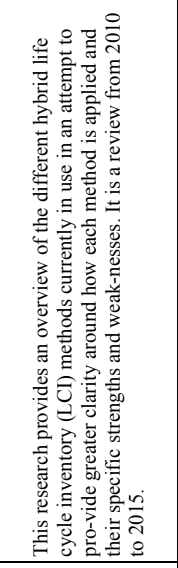 & 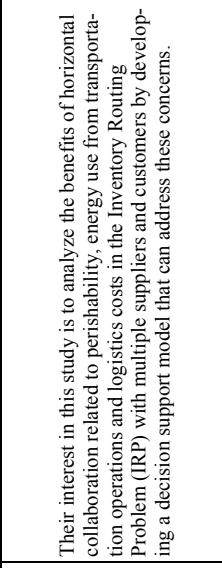 \\
\hline Publication year & 2018 & 2018 \\
\hline $\begin{array}{c}\text { The first author } \\
\text { name }\end{array}$ & $\begin{array}{l}\text { Crawford and } \\
\text { et al. }\end{array}$ & Soysal and et al. \\
\hline Author No. & 趈 & $\sqrt[n]{n}$ \\
\hline
\end{tabular}


Table 2. Distribution of researches based on the name of journals.

\begin{tabular}{|c|c|c|c|}
\hline No. & The name of Journals & $\begin{array}{c}\text { Frequency of Articles } \\
\text { are Published }\end{array}$ & $\begin{array}{c}\text { Percent- } \\
\text { age }\end{array}$ \\
\hline 1 & Systems Engineering Procedia & 1 & $2 \%$ \\
\hline 2 & Journal of Operations Management & 1 & $2 \%$ \\
\hline 3 & Social and Behavioral Sciences & 1 & $2 \%$ \\
\hline 4 & $\begin{array}{l}\text { Journal of Industrial Engineering Research in Production Sys- } \\
\text { tems }\end{array}$ & 1 & $2 \%$ \\
\hline 5 & Advances in decision sciences & 1 & $2 \%$ \\
\hline 6 & Science of The Total Environment & 1 & $2 \%$ \\
\hline 7 & Engineering Applications of Artificial Intelligence & 1 & $2 \%$ \\
\hline 8 & Journal of Resources Policy & 1 & $2 \%$ \\
\hline 9 & Technological Forecasting and Social Change & 1 & $2 \%$ \\
\hline 10 & Safety Science & 1 & $2 \%$ \\
\hline 11 & Journal of Loss Prevention in the Process Industries & 1 & $2 \%$ \\
\hline 12 & Asia Pacific Management Review & 1 & $2 \%$ \\
\hline 13 & Journal of Manufacturing Systems & 1 & $2 \%$ \\
\hline 14 & Future Business Journal & 1 & $2 \%$ \\
\hline 15 & Science Direct Procedia CIRP & 1 & $2 \%$ \\
\hline 16 & Knowledge-Based Systems & 1 & $2 \%$ \\
\hline 17 & Journal of Optimization in Industrial Engineering & 1 & $2 \%$ \\
\hline 18 & European Journal of Operational Research & 1 & $2 \%$ \\
\hline 19 & IFAC Paper Online & 1 & $2 \%$ \\
\hline 20 & Journal of Reliability Engineering and System Safety & 1 & $2 \%$ \\
\hline 21 & Computers \& Industrial Engineering & 2 & $4 \%$ \\
\hline 22 & Journal of Omega & 2 & $4 \%$ \\
\hline 23 & Journal of computers and Chemical Engineering & 2 & $4 \%$ \\
\hline 24 & Applied Mathematical Modelling & 2 & $4 \%$ \\
\hline 25 & Journal of Computers and Operations Research & 2 & $4 \%$ \\
\hline 26 & Journal of Industrial and Systems Engineering & 3 & $5 \%$ \\
\hline 27 & Journal of Transportation Research Part E & 3 & $5 \%$ \\
\hline 28 & Expert Systems with Applications & 4 & $6 \%$ \\
\hline 29 & Journal of Cleaner Production & 5 & $9 \%$ \\
\hline \multirow[t]{2}{*}{30} & Int. J. of Production Economics & 11 & $20 \%$ \\
\hline & Total & 56 & $100 \%$ \\
\hline
\end{tabular}

Table 3. Distribution of researches based on the countries.

\begin{tabular}{|c|c|c|}
\hline No. & The name of the Country & Percentage \\
\hline 1 & Spain & $1 \%$ \\
\hline 2 & Italy & $1 \%$ \\
\hline 3 & Poland & $1 \%$ \\
\hline 4 & Norway & $1 \%$ \\
\hline 5 & Ecuador & $1 \%$ \\
\hline
\end{tabular}




\begin{tabular}{|c|c|c|}
\hline 6 & Netherland & $1 \%$ \\
\hline 7 & Australia & $1 \%$ \\
\hline 8 & Denmark & $1 \%$ \\
\hline 9 & Minnesota & $1 \%$ \\
\hline 10 & New Zealand & $3 \%$ \\
\hline 11 & France & $3 \%$ \\
\hline 12 & Turkey & $3 \%$ \\
\hline 13 & Switzerland & $3 \%$ \\
\hline 14 & Sweden & $3 \%$ \\
\hline 15 & India & $4 \%$ \\
\hline 16 & Germany & $4 \%$ \\
\hline 17 & Brazil & $4 \%$ \\
\hline 18 & Malaysia & $4 \%$ \\
\hline 19 & UK & $14 \%$ \\
\hline 20 & US & $17 \%$ \\
\hline 21 & Iran & $24 \%$ \\
\hline 22 & China & $\mathbf{1 0 0 \%}$ \\
\hline & & Total \\
\hline
\end{tabular}

Table 4. Distribution of researches based on the methods.

\begin{tabular}{|c|l|c|c|}
\hline No. & \multicolumn{1}{|c|}{ The name of methods } & Methods frequency & Methods percentage \\
\hline 1 & Peak value method of extreme value theory & 1 & $2 \%$ \\
\hline 2 & Vignette-based field experiment & 1 & $2 \%$ \\
\hline 3 & Two-stage game & 1 & $2 \%$ \\
\hline 4 & EOQ Model & 1 & $2 \%$ \\
\hline 5 & RL algorithm & 1 & $2 \%$ \\
\hline 6 & Markov chain & 1 & $2 \%$ \\
\hline 7 & Two-stage ordering policy & 1 & $2 \%$ \\
\hline 8 & Typical example & 1 & $2 \%$ \\
\hline 9 & GSCM optimization Model & 1 & $2 \%$ \\
\hline 10 & GABC algorithm & 1 & $2 \%$ \\
\hline 11 & Parallel LaGrange algorithm & 1 & $2 \%$ \\
\hline 12 & Two-stage programming & 1 & $2 \%$ \\
\hline 13 & D-invariance properties & 1 & $2 \%$ \\
\hline 14 & Multi-echelon Model & 1 & $2 \%$ \\
\hline 15 & Uncertainty programming & 1 & $2 \%$ \\
\hline 16 & Robust optimization & 1 & $2 \%$ \\
\hline 17 & Newsvendor-type item method & 1 & $2 \%$ \\
\hline 18 & Statistical model & 1 & $2 \%$ \\
\hline 19 & Dynamic programming & 1 & $2 \%$ \\
\hline 20 & EPQ Model & 1 & $2 \%$ \\
\hline 21 & Horizontal collaboration model & 2 & \\
\hline 22 & Game theory & & $2 \%$ \\
\hline & & 1 & \\
\hline
\end{tabular}




\begin{tabular}{|c|l|c|c|}
\hline 23 & ABC algorithm & 2 & $4 \%$ \\
\hline 24 & Regression & 2 & $4 \%$ \\
\hline 25 & Bayesian networks & 2 & $4 \%$ \\
\hline 26 & Numerical Model & 2 & $4 \%$ \\
\hline 27 & Genetic algorithm & 2 & $4 \%$ \\
\hline 28 & Activity based costing model & 2 & $4 \%$ \\
\hline 29 & Fuzzy logic & 3 & $5 \%$ \\
\hline 30 & Non-linear programming & 3 & $5 \%$ \\
\hline 31 & Multi-stage stochastic programming & 3 & $5 \%$ \\
\hline 32 & Mathematical Model & 4 & $7 \%$ \\
\hline 33 & Simulation & 4 & $7 \%$ \\
\hline 34 & Heuristic algorithm & 4 & $7 \%$ \\
\hline & & $\mathbf{5 6}$ & $\mathbf{1 0 0} \%$ \\
\hline
\end{tabular}

Table 5. Distribution of researches based on the regions

\begin{tabular}{|c|c|c|}
\hline The name of the regions & Number & Percentage \\
\hline Australia & 2 & $8 \%$ \\
\hline Asia & 4 & $17 \%$ \\
\hline America & 7 & $29 \%$ \\
\hline Europe & 11 & $46 \%$ \\
\hline Total & 24 & $100 \%$ \\
\hline
\end{tabular}

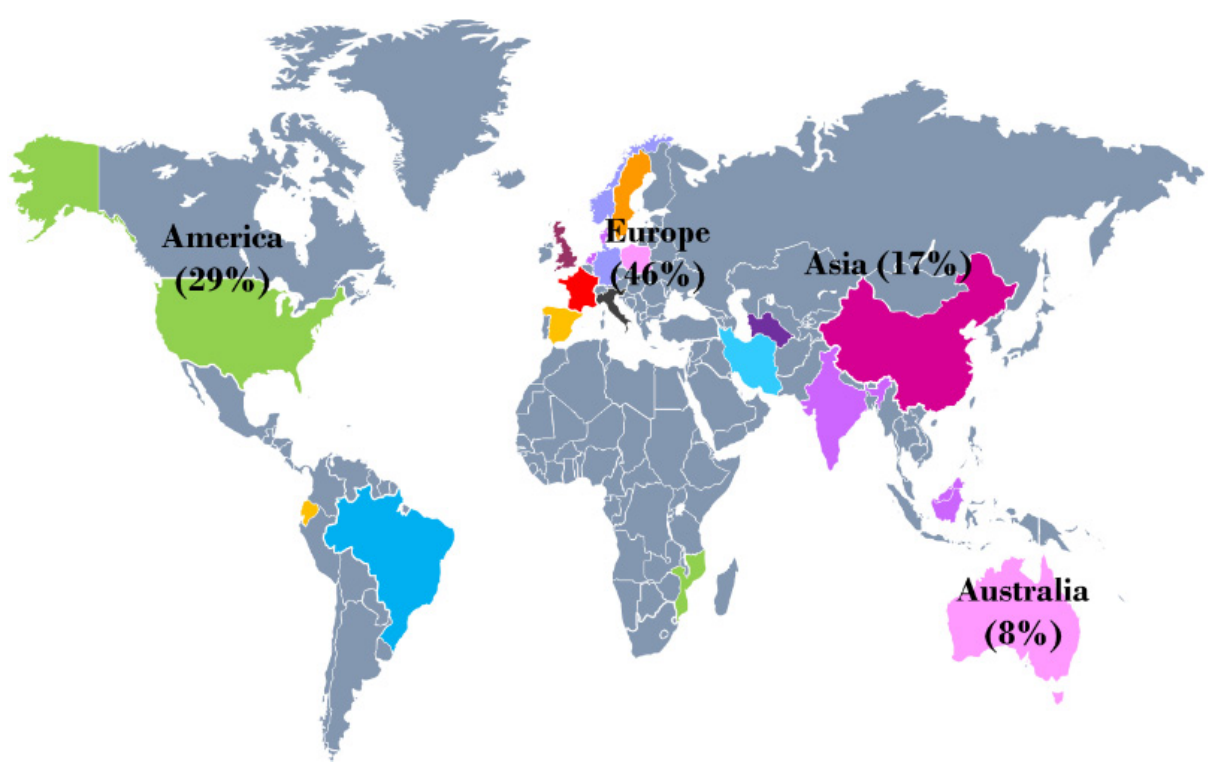

Fig. 2. Distribution of researches based on regions. 


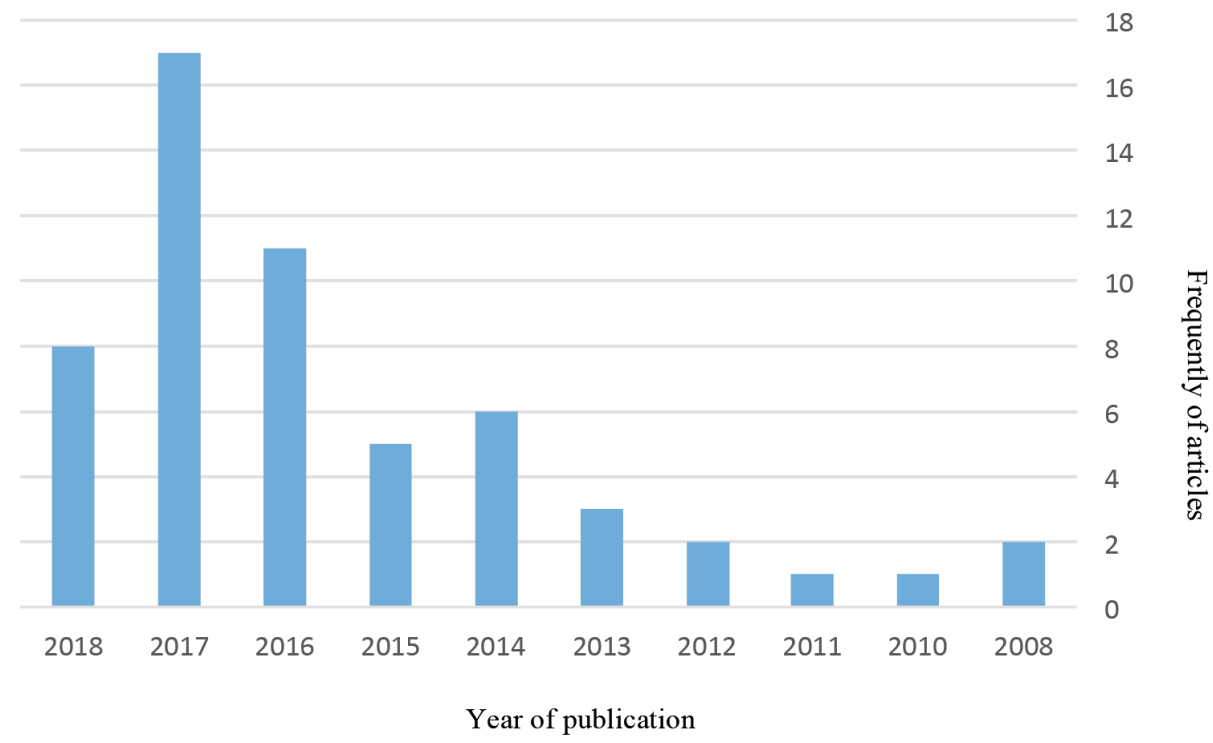

Fig. 3. Distribution of researches based on year of publication.

\section{Discussion}

Order management and inventory control also their costs are problems that often puzzles managers and researchers. An enormous range of factors include re-ordering point, operational risk, lead time, demand and pricing risk, product innovation, reduced collection demands and documentary credits alongside order management and inventory control. Pricing risk, product innovation, reduced collection demands and documentary credits are critical factors that have not studied yet. In this paper, as a result of distribution of researches based on the countries which the highest contribution was from China (see Table 3). Europe continent has had the largest participation in this field (see Table 5 and Fig. 2). Regarding to journals distribution, International Journal of Production Economics was ranked as the first journal as exposed in Table 2. Some manufacturing industries that are surveyed in articles as a case study or collocated data from them, comprise bicycle, nylon plastic and refrigerator manufacturing, façade components, the carpet manufacturing facility, automotive supply chain, coal mining enterprises, gas industry, steel and glass company, locomotives railways and energy production units but other manufacturing industries which have not checked out yet such as smartphone plant, clothing producers, paint factory, furniture manufacturing and so on. Likewise, the methods that are used more than others consist mathematical model, simulation and heuristic algorithm that the applications of artificial neural network, ant colony algorithm and queue theory, still have not probed (Table 4). Distribution of researches based on year of publication, maximum number of articles which were published is in 2017 by 17 articles as well as, minimum number of articles which were published is in 2010 by an article (Fig. 3). 


\section{Conclusion \& Recommendation}

This review paper aimed to review previous studies that applied order management and inventory control during 2008 until 2018 in 32 international scholarly journals which are indexed in Science direct and Scopus databases. In addition, this review paper classifies published articles into 8 main areas: the author number, The first author name, publication year, problems, factors, type of manufacturing industries, research methods and results and findings. As a result of probing 56 articles, 6 articles have implemented ABC approaches, nevertheless, the need for further research is felt in setting of inventory and order of the means of production that not considered synchronously. Moreover, a number of factors for instance product innovation, reduced collection demands and documentary credits are important too that have not surveyed yet. Based on review findings, research methods for instance the applications of artificial neural networks, ant colony algorithm and queue theory have not studied also, we recommended them for future researches. This review paper classified the selected articles into 8 fields of order management and inventory control, it is suggested that future researches can review and classify articles in different areas and sub-areas. This review paper checks out articles that are published in Science direct and Scopus databases, thus, future review researches can peruse other databases. In addition; our review paper only focused on English scholarly journals rather than other languages, therefore; future review surveys can consider and focus on other languages. Because of the importance and necessity of researching in order and inventory, it is recommended that in the fields of operational risk, the collection of demands and documentary credits along with $\mathrm{ABC}$ approach come to future studies. It is also suggested that a combination model of these four items will be presented. These fields mentioned above are critical to answer the needs and orders of all customers as well as the survival of an organization, which if ignored, the organization will face with a lack of funds and, in consequence, a bankruptcy and elimination of the competition cycle completely will come about. Moreover, it is recommended that further researches will be done in the fields mentioned with different state of multi-product, single-product or perishable products and green supply chain, also their costs or the closed-loop supply chain with two forward and backward flows to help managers in costs managing of their supply and demand and organization's survival.

\section{$5 \quad$ References}

[1] Berling, P. (2008). Holding cost determination: An activity-based cost approach. Int. J. of Production Economics, 112: 829-840 https://doi.org/10.1016/j.ijpe.2005.10.010

[2] Charles, S. L. and Hansen, D. R. (2008). An evaluation of activity-based costing and functional-based costing: A game-theoretic approach. Int. J. of Production Economics, 113: 282-296 https://doi.org/10.1016/j.ijpe.2007.08.008

[3] Askarany, D., Yazdifar, H. and Askary, S. (2010). Supply chain management, activitybased costing and organizational factors. Int. J. Production Economics, 127: 238-248 https://doi.org/10.1016/j.ijpe.2009.08.004 
[4] Haji, R., Haji, A. and Safari, M. (2011). Queuing Inventory System in a Two-level Supply Chain with One-for-One Ordering Policy. Journal of Industrial and Systems Engineering, 5: $52-62$

[5] Feng-gea, Y. and Ping, Z. (2012). The Measurement of Operational Risk Based on CVaR: A Decision Engineering Technique. Systems Engineering Procedia, 4: 438-447 https://doi.org/10.1016/j.sepro.2012.01.008

[6] Schulze, M., Seuring, S. and Ewering, C. (2012). Applying activity-based costing in a supply chain environment. Int. J. Production Economics, 135: 716-725 https://doi.org/10.1016/j.ijpe.2011.10.005

[7] Hora, M. and Klassen, R. D. (2013). Learning from others' misfortune: Factors influencing knowledge acquisition to reduce operational risk. Journal of Operations Management, 31: 52-61 https://doi.org/10.1016/j.jom.2012.06.004

[8] Alinezhad Sarokolaei, M., Saviz, M., Fathi Moradloo, M. and Soleimani Dahajd, N. (2013). Time Driven Activity based Costing by Using Fuzzy Logics. Social and Behavioral Sciences, 75: 338-345 https://doi.org/10.1016/j.sbspro.2013.04.038

[9] Wei, Y., Hu, Q. and Xu, C. (2013). Ordering, pricing and allocation in a service supply chain. Int. J. Production Economics, 144: 590-598 https://doi.org/10.1016/j.ijpe.2013 .04 .022

[10] Tat, R., Ismaili, M. and Taleizadeh, A. (2014). Developing EOQ model with instantaneous deteriorating items for a vendor-managed inventory (VMI) system. Journal of Industrial and Systems Engineering, 7: 21-42

[11] Baradaran Kazemzadeh, R., Kaheh, Z. and Masehian, E. (2014). A Mixed Integer Nonlinear Programming Model for Order Replenishment and a Heuristic Algorithm for its Solution. Journal of Industrial Engineering Research in Production Systems, 2: 63-75

[12] Nooraie, S. and Mellat Parast, M. (2014). A Multi-Objective Approach to Supply Chain Risk Management: Integrating Visibility with Supply and Demand Risk. Int. J. of Production Economics: Manufacturing Systems, Strategy \& Design, 161: 192-200 https://doi.org/10.1016/j.ijpe.2014.12.024

[13] Xiao, T., Shi, J. and Chen, G. (2014). Price and lead time competition, and coordination for make-to-order supply chains. Computers \& Industrial Engineering, 68: 23-34 https://doi.org/10.1016/j.cie.2013.11.015

[14] Molamohamadi, Z., Arshizadeh, R., Ismail, N. and Azizi, A. (2014). An Economic Order Quantity Model with Completely Backordering and No Decreasing Demand under TwoLevel Trade Credit. Advances in decision sciences, SCOPUS, 2014: 1-11 https://doi.org/10.1155/2014/340135

[15] Bieda, B. (2014). Application of stochastic approach based on Monte Carlo (MC) simulation for life cycle inventory (LCI) to the steel process chain: Case study. Science of The Total Environment, 481: 649-655 https://doi.org/10.1016/j.scitotenv.2013.10.123

[16] Mohammad dust, F., Reza pour, S., Zanjirani Farahani, R., Mofidfar, M. and Hill, A. (2015). Developing lean and responsive supply chains: A robust model for alternative risk mitigation strategies in supply chain designs. Int. J. of Production Economics: Manufacturing Systems, Strategy \& Design, 183: 21-59

[17] Guertler, B. and Spinler, S. (2015). When does Operational Risk Cause Supply Chain Enterprises to Tip? A Simulation of Intra-Organizational Dynamics. Journal of Omega, 57: 54-69 https://doi.org/10.1016/j.omega.2015.03.005

[18] Mortazavi, A., Arshadi Khamseh, A. and Azimi, P. (2015). Designing of an intelligent self-adaptive model for supply chain ordering management system. Engineering Applications of Artificial Intelligence, 37: 207-220 https://doi.org/10.1016/j.engappai.2014.09.004 
[19] Zhang, L., Lee, C. and Zhang, S. (2015). An Integrated Model for Strategic Supply Chain Design: Formulation and ABC-based Solution Approach. Expert Systems with Applications, 52: 12-36

[20] Jiang, Y., Chen, M. and Zhou, D. (2015). Joint optimization of preventive maintenance and inventory policies for multi-unit systems subject to deteriorating spare part inventory. Journal of Manufacturing Systems, 35: 191-205 https://doi.org/10.1016/j.jmsy.2015. $\underline{01.002}$

[21] Dhegihan, E., Behfar, N. and Jabalameli, M. (2016). Optimizing location, routing and inventory decisions in an integrated supply chain network under uncertainty. Journal of Industrial and Systems Engineering, 9: 93-111

[22] Zhang, S., Lee, C., Wu, K. and Choy, K. (2016). Multi-objective optimization for sustainable supply chain network design considering multiple distribution channels. Expert Systems with Applications, 65: 87-99 https://doi.org/10.1016/j.eswa.2016.08.037

[23] Nawrocki, T. L. and Jonek-Kowalska, I. (2016). Assessing operational risk in coal mining enterprises - Internal, industrial and international perspectives. Journal of Resources Policy, 48: 50-67 https://doi.org/10.1016/j.resourpol.2016.02.008

[24] Yousefi Babadia, A., Tavakkoli Moghaddam, R., Bozorgi Amiria, A. and Saiph, S. (2016). Designing a Reliable Multi-Objective Queuing Model of a Petrochemical Supply Chain Network under Uncertainty: A Case Study. Journal of computers and Chemical Engineering, 100: 1-61

[25] Zhang, Q., Zhang, D., Tsao, Y. and Luo, J. (2016). Optimal ordering policy in a two-stage supply chain with advance payment for stable supply capacity. Int. J. Production Economics, 177: 34-43 https://doi.org/10.1016/j.ijpe.2016.04.004

[26] Zhao, C., Song, H. and Chen, W. (2016). Can social responsibility reduce operational risk: Empirical analysis of Chinese listed companies. Technological Forecasting and Social Change, 112: 145-154 https://doi.org/10.1016/j.techfore.2016.08.023

[27] Yang, X. and Haugen, S. (2016). Risk information for operational decision-making in the offshore oil and gas industry. Safety Science, 86: 98-109 https://doi.org/10.1016/j.ssci. 2016.02.022

[28] Barua, S., Gao, X., Pasman, H. and Mannan, M.S. (2016). Bayesian network based dynamic operational risk assessment. Journal of Loss Prevention in the Process Industries, 41: 399-410 https://doi.org/10.1016/j.jlp.2015.11.024

[29] Zhang, X., Huang, S. and Wan, Z. (2016). Optimal pricing and ordering in global supply chain management with constraints under random demand. Applied Mathematical Modelling, 40: 10105-10130 https://doi.org/10.1016/j.apm.2016.06.054

[30] Wang, K. H., Huang, Y. Ch. and Tung, Ch. T. (2016). A return-policy contract with a stock-dependent demand and inventory shrinkages. Asia Pacific Management Review, 21: 154-160 https://doi.org/10.1016/j.apmrv.2016.02.001

[31] Elsayedn, Kh. and Wahba, H. (2016). Reexamining the relationship between inventory management and firm performance: An organizational life cycle perspective. Future Business Journal, 2: 65-80 https://doi.org/10.1016/j.fbj.2016.05.001

[32] Cui, Y., Guan, Z., Saiph, U., Zhang, L., Zhang, F. and Mirza, J. (2017). Close Loop Supply Chain Network Problem with Uncertainty in Demand and Returned Products: Genetic Artificial Bee Colony Algorithm Approach. Journal of Cleaner Production, 17: 27-65

[33] Lu, T. Y., Wang, S., Wuc, M. and Cheng, F. (2017). Competitive Price Strategy with Activity-Based Costing: Case Study of Bicycle Part Company. Science Direct Procedia CIRP, 63: 14-20 https://doi.org/10.1016/j.procir.2017.03.102

[34] Fattahi, M., Govindan, K. and Keyvanshokooh, E. (2017). Responsive and resilient supply chain network design under operational and disruption risks with delivery lead-time sensi- 
tive customers. Transportation Research Part E, 101: 176-200 https://doi.org/10.1016/j.tre. 2017.02.004

[35] Pacheco, E. d. O., Canella, S., Lüders, R., Paula, A. and Póvoa, B. (2017). Order-up-tolevel Policy Update Procedure for a Supply Chain Subject to Market Demand Uncertainty. Computers \& Industrial Engineering, 113: 347-355 https://doi.org/10.1016/j.cie.2 $\underline{017.09 .015}$

[36] Xu, X., Zhang, W., He, P. and Xu, X. (2017). Production and pricing problems in make-toorder supply chain with cap-and-trade regulation. Journal of Omega, 66: 248-257 https://doi.org/10.1016/j.omega.2015.08.006

[37] Nematollahia, M., Hosseini-Motlagha, S. and Heydari, J. (2017). Coordination of social responsibility and order quantity in a two-echelon supply chain: A collaborative decisionmaking perspective. Int. J. Production Economics, 148: 107-121 https://doi.org/10.1016/ j.ijpe.2016.11.017

[38] Li, J., Zeng, X., Liu, C. and Zhou, X. (2017). A parallel Lagrange algorithm for order acceptance and scheduling in cluster supply chains. Knowledge-Based Systems, In press

[39] Kumar, J., Roy, N., Mostafaei pour, A. and Qolipour, M. (2017). Development of a Novel Lot-sizing Model with Variable Lead Time in Supply Chain Environment. Journal of Optimization in Industrial Engineering, 22: 25-38

[40] Govindan, K., Fattah, M. and Keyvan shokooh, E. (2017). Supply chain network design under uncertainty: A comprehensive review and future research directions. European Journal of Operational Research, 263: 1-49 https://doi.org/10.1016/j.ejor.2017.04.009

[41] Arampantzi, C. and Minis, L. (2017). A new model for designing sustainable supply chain networks and its application to a global manufacturer. Journal of Cleaner Production, 17: 4-64 https://doi.org/10.1016/j.jclepro.2017.03.164

[42] Vahidi, F., Torabi, S.A. and Ramezankhani, M.J. (2018). Sustainable supplier selection and order allocation under operational and disruption risks. Journal of Cleaner Production, 174: 1351-1365 https://doi.org/10.1016/j.jclepro.2017.11.012

[43] Wanke, P., Alvarenga, H., Correa, H., Vencheh, A. H. and Azad, A. K. (2017). Fuzzy inference systems and inventory allocation decisions: Exploring the impact of priority rules on total costs and service levels. Journal of Expert Systems with Applications, 85: 182-193 https://doi.org/10.1016/j.eswa.2017.05.043

[44] Abbou, R., Loiseau, J. J., Khaldi, H. and Farra, B. B. (2017). On Inventory Control for Perishable Inventory Systems Subject to Uncertainties On Customer Demands. IFAC Paper Online, 50: 10172-10177 https://doi.org/10.1016/j.ifacol.2017.08.1765

[45] Li, M. and Wang, Z. (2017). An Integrated Replenishment and Production Control Policy under Inventory Inaccuracy and Time-delay. Journal of Computers and Operations Research, 88; 137-149 https://doi.org/10.1016/j.cor.2017.06.014

[46] Moser, Ph., Isaksson, O. and Seifert, R. W. (2017). Inventory dynamics in process industries: An empirical investigation. Int. J. of Production Economics, 191: 253-266 https://doi.org/10.1016/j.ijpe.2017.06.019

[47] Marand, A. J., Li, H. and Thorstenson, A. (2017). Joint inventory control and pricing in a service-inventory system. Int. J. of Production Economics, In press

[48] Daia, Z., Alana, F. and Gaoc, K. (2017). Optimizing multi-echelon inventory with three types of demand in supply chain. Journal of Transportation Research Part E, 107: 141-177 https://doi.org/10.1016/j.tre.2017.09.008

[49] Taleizadeh, A. A., Soleymanfar, V. R. and Govindan, K. (2018). Sustainable economic production quantity models for inventory systems with shortage. Journal of Cleaner Production, 174: 1011-1020 https://doi.org/10.1016/j.jclepro.2017.10.222 
[50] Sýkora, M., Markova, J. and Diamantidis, D. (2018). Bayesian network application for the risk assessment of existing energy production units. Journal of Reliability Engineering and System Safety, 169: 312-320 https://doi.org/10.1016/j.ress.2017.09.006

[51] Rafie-Majd, Z., Pasandideh, H. R. and Naderi, B. (2018). Modelling and Solving the Integrated Inventory-Location-Routing Problem in a multi-period and multi-perishable Product Supply Chain with Uncertainty: Lagrangian Relaxation Algorithm. Journal of Computers and Chemical Engineering, 109: 9-22 https://doi.org/10.1016/j.compchemeng. 2017.10.013

[52] Liu, W., Wu, R., Liang, Z. and Zhu, D. (2018). Decision model for the customer order decoupling point considering order insertion scheduling with capacity and time constraints in logistics service supply chain. Applied Mathematical Modelling, 54: 112-135 https://doi.org/10.1016/j.apm.2017.09.027

[53] Kara, A. and Dogan, I. (2018). Reinforcement learning approaches for specifying ordering policies of perishable inventory systems. Journal of Expert Systems with Applications, 91: 150-158 https://doi.org/10.1016/j.eswa.2017.08.046

[54] Crawford, R. H., Bontinck, P. A., Stephan, A., Wiedmann, T. and Yu, M. (2018). Hybrid life cycle inventory methods - A review. Journal of Cleaner Production, 172: 1273-1288 https://doi.org/10.1016/j.jclepro.2017.10.176

[55] Soysal, M., Bloemhof Ruwaard, J. M., Haijema, R. and van der Vorst, J. G.A.J. (2018). Modeling a green inventory routing problem for perishable products with horizontal collaboration. Journal of Computers and Operations Research, 89: 168-182 https://doi.org/10.1016/j.cor.2016.02.003

[56] Zhalechiana, M., Ali Torabib, S. and Mohammadi, M. (2018). Hub-and-spoke network design under operational and disruption risks. Transportation Research Part E, 109: 20-43 https://doi.org/10.1016/j.tre.2017.11.001

\section{Authors}

Zohreh Momeni is master Industrial Management student in Science and Research Branch Islamic Azad University of Tehran, Iran.

Amir Azizi is Faculty member in Industrial Engineering department in Science and Research Branch Islamic Azad University of Tehran, Iran. azizi@srbiau.ac.ir

Article submitted 02 December 2017. Final acceptance 22 April 2018. Final version published as submitted by the authors. 